Historic, Archive Document

Do not assume content reflects current scientific knowledge, policies, or practices. 


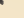




\section{CATALOGUE '29}

\section{FLOWERING BULBS}

FOR YOUR GARDEN

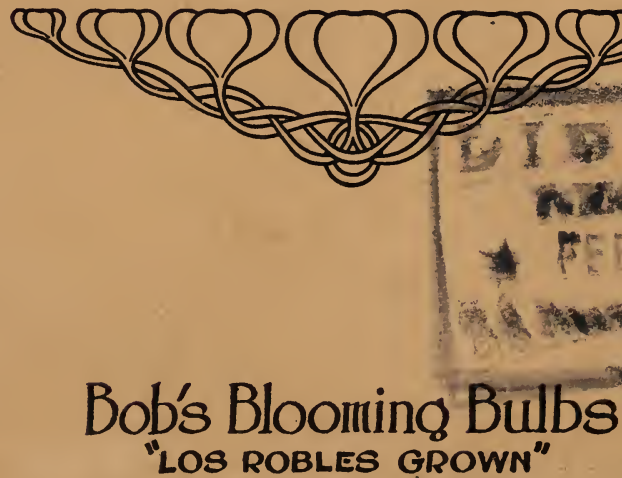

\section{Gladiol GARDENS "THE LINCOLN WAY" \\ ON THE EAST CLIFF DRIVE, Santa Cruz Cal.}




\section{OUR POLICY OF SERVICE}

Our SERVICE POLICY in merchandising does not mean just courteous salesmanship, prompty delivery, good measure, and the like.

It consists of a great deal more than those-service in ascertaining and fulfilling the needs and desires of the public in a satisfactory and economical manner, the searching out of the best markets of the world from which may be procured the products best suited to meet our customers' requirements; merchandise which must meet and conform to the very highest standards, and which must also represent the maximum of value that is possible for us to offer at any fair price.

An old Persian proverb says, "The Priceless Ingredient of any product in the world is the Honor, and the Integrity of him who makes it!"

The safe delivery of our merchandise to a customer is never the conclusion of a transaction with us. We are interested in that customer for all time, for in the merchandise he receives is built all the Honor and the Integrity it is possible to include in any product or transaction!

By a close intimacy with the fundamentals of business ethics, by an intimate supervision of the many details involved in growing and marketing, and by a never-ending effort and interest in making our bulbs satisfactory to our customers, we are ever striving to better serve those who purchase from us.

For us, the growing of bulbs is not alone a business. In it we find a rare pleasure which compensates for many of the disappointments and hardships which come in any commercial enterprise. It is doubtful if ever there was a human being with high instincts, who ever was tired by the endless variety, and the glorious colorings of the bulbous flowers.

We would not find this pleasure in business were we to give each buyer just his money's worth of what his order called for, and then call the transaction finished!

Our pleasure is largely found in your pleasure-in the enjoyment you find in your flowers which have come to you from us, and in the delivery of that intangible something called "Good Will" or "Service," and which is always included in the delivery of our orders, "No Charge."

We will continue in our efforts to Serve, for a life-time of willing Service has made us incomparable Friendships of incalculable value. 


\section{TERMS}

CASH.

Six bulbs at the dozen rate

Fifty at the hundred rate

We prepay delivery charges

\section{REMITTANCES}

Customer's personal check preferred and will be customer's receipt. Any other form accepted.

\section{DISCLAIMER}

Most of the failures with seeds and bulbs are due to causes entirely beyond our control, such as unfavorable weather, too deep or too shallow planting, slugs, etc.; therefore we give no warranty, expressed or implied, as to description, purity, productiveness, or any other matter of any seeds, plants, bulbs, etc., we send out, and will in no way be responsible for the crop; if the purchaser does not accept the goods on these terms they are to be returned at once.

\section{SHIPPING SEASONS}

The shiping seasons are of course dependent upon the range of the planting season. It is advisable to order EARLY and we will deliver as the stock is ready to plant.

\section{INSPECTION LAWS}

All stocks are shipped under the supervision of the inspectors of the County Horticultural Commissioner, subject to final passing by your County Commissioner. All stock shipped is supposed to be free from all insects and disease. In case stock should for any reason be condemned by your Commissioner, we will gladly refund the money. That is all we can do. 


\section{N D E X}

AGAPANTHUS ....... 53

AMARYLLIS ....... 52

ANEMONES . . . . . . 52

Seed .......... 52

ALSTROMERIA ....... 52

BABIANA ........ 55

BEGONIAS . ....... 55

CALLAS .......... 51

CROCUS ......... 36

DAFFODILS ......... 35

FLOWER SEEDS . . . . 59-69

FREESIAS ........54

FERTILIZERS . . . . . . 70

GLADIOLI . . . . . . . . 3-29

Gift Boxes . ...... 31

Mixtures ........29

Collections .......32

Seed . . . . . . . . 32

Miniature ....... 34

Butterfly .......... 30

GIFT BOXES ......... 31

GOPHER GUNS . . . . . 70

GLOXINIAS . . . . . . 54

HYACINTHS . . , , . 36

IRIS-

Spanish .......442

Holland . . . . . . . 43

German ....... 44-47

Stylosa . . ...... 47

Susiana ........ 43

Orientalis .......448

Japonica . . . . . . . 48

Pavonia ..........44

IXIAS . . . . . . . . 55

LILIES
LAPEYROUSIA ....... 58

MONTBRETIAS . . . . . 56

MUSCARI ......... 55

NARCISSUS ....... 35

NERINE . . . . . . . 56

OXALIS ......... 56

RANUNCULUS ....... 53

SNOWFLAKES . . . . . . 55

SPARAXIS ........ 58

SCILLAS ............... SEEDS-

Antirhinnum ..... 5 59

Aster ........660

Delphinium ......661

Stochs . . . . 6 62-63

Sweet Peas . . . . 63-66

Zinnias ...... 66-68

Grass ........669

Anemone ....... 52

Gladioli . . . . . . . 32

Marigold . . . . . 61-62 TULIPS-

Darwin ....... 37-3s

Breeder . . . . . . 29 29

Rembrandt ......440

Mendel .........41

Cottage ........44

Parrott ........ 42

Collections ........39

Striped .........44 41

Lily Flowered . ..... 42

TRITONIA .......... 5 T

TRITELIA .......... 5 T

WATSONIA ........ 57

ZEPHRYANTES ...... 58

\section{PLACE YOUR ORDER FOR BULBS NOW!}

Deliveries will be made as soon as stock is ready to plant This insures your planting at the right season. 


\section{GLADIOLI}

With the exception of questions concerning culture, there is no question more frequently asked than the correct pronunciation of the word, gladiolus. As the official pronunciation is changed with every change of the moon, Dr. Bales of Triangle Farm, Circleville, Ohio, prompted to pen the following lines of doggerel:

Some folks call me glad-dee'-olus, (from gladius, meaning sword) And some call me glad-die'-olus, a dictionary word.

Some call be gladi-o'-lus, which really is not bad,

But when I bloom my very best, both you and I'll be GLAD.

\section{GLADIOLUS CULTURE}

Although no flower gives more satisfaction than the Gladiolus, with ordinary care and culture in average soils, yet it readily responds to high culture, and the superior blooms produced by special attention amply repay for the additional care. Only those who have seen the Gladiolus at its best can realize the vast difference between the blossoms produced by first-class methods and those less carefully grown. We strongly advise our friends to employ only the best cultural methods possible.

It is advantageous to spade in a good covering of manure late in the fall preceding the plantings. The richer the soil and better the care and culture the better the results will be. In the spring, when the soil is in proper condition, spade deeply and pulverize thoroughly. Fresh manure should not be used. Select a sunny location. "Glads" are like folks-they need a lot of sunshine or they appear pale and anemic and sickly.

For a long blooming season, plant at intervals of about two weeks from the first garden-making time until the middle or latter part of June. Bulbs can be planted four inches deep and over in mellow soils, and four to eight inches apart in the row. The deeper planting will help to keep the plants from falling over at blooming time.

Frequent thorough waterings of the soil in the beds at evening is a wonderful stimulant and gives the blooms much greater sun resistance and lasting qualities. Keep the soil well worked, especially early the next forenoon after being watered the previous evening. Always keep a soil crust from forming in the rows among the plants. After blooming spikes begin to show, cultivate only about two inches deep. Do not work the soil when wet. Do not plant too near to walls or shrubbery.

Varieties vary in different soils and seasons. Some do their best only in alternate years. Some varieties produce monster bulbs 
while others produce small ones. The bulbs of most varieties have a fine satiny covering, but some have a rough husk.

Slender stakes of wood or bamboo painted a light green make good supports for the spikes at blooming time. Where horizontal lines are used they should be fastened to end posts firmly set and the wires tightly stretched.

If wanted for cut-flower purposes, the flower-spikes should be cut when the first bloom opens. Four leaves of the plant should remain to properly mature the bulb. After cutting the flower-spike, place it in water. One bud after another will open. Each morning cut off the stem on a long slant, remove the withered flowers and give fresh water. Drooping flowers will revive, if placed in a cool cellar over night.

Dig bulbs during October; cut off the top close to the bulb and cure by drying a few days in or out-of-doors, then store away in boxes not over 4 inches deep in a cool part of a frost-proof cellar. Bulbs should not be kept in air-tight packages.

\section{GLADIOLI}

Large 11/4 Inch Up

ALICE

\section{Medium 1 inch}

ea.
Small $1 / 2$ inch diam.

6

1915)-Large, orange, saffron

flowers of hybrid Prim type.

Will not fade in color. Very fine.

Large............
Medium........
Small.........
shell pink

AMERICA-Beautiful shell pink
with lavender suffusion over entire flower.

\section{Large \\ Medium \\ Small}

ANNA EBERIUS (Diener) Velve-

ty, lavender purple with darker center.

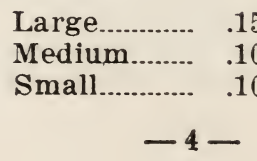

$\begin{array}{rrrrrr}.10 & \$ .30 & \$ .50 & \$ 1.00 & \$ 6.00 \\ .10 & .20 & .30 & .60 & 4.00 \\ .05 & .10 & .15 & .30 & 2.00\end{array}$


A. B. KUNDRED (K-1918). Tall ea.

3

6

12

100 large, intensely ruffled flower, delicate deep cream, with pink and yellow markings

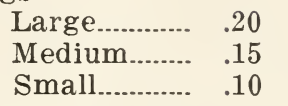
.55

Small...........

ARIZONA-Dark pink with maroon markings on lower petals

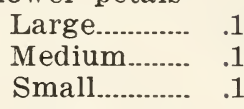

$\begin{array}{rr}1.25 & 7.00 \\ .75 & 5.00 \\ .50 & 3.00\end{array}$

AURORA-(Velthys 1916). In-

tense scarlet. Early

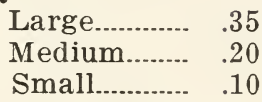

AUGUSTA-Pale white, blue an-

ther

Large...........
Medium........

Small

ATTRACTION - Fin e scarlet, white feathered throat.

Large.............
Medium.......
Small............
l ruffled va-

ALTON-A wonderful ruffled
riety of finest orange color

Large...

Medium

Small
.10

.10
1.00

.55

.25

1.75

1.00

.40

.25

.15

.40

.25

.10
3.50

2.00

.75

.75

.50

25

.75

.50

.25

.10

.25

.15
.40

.25

AUTUMN QUEEN-Light yel.20

.15

.55

.35

.25

1.00

.65

.40

2.00

1.25

.75

5.00

3.00

1.50

low with red blotch in throat.

Late bloomer

Large...........
Medium.......
Small...........
ty) A beau-

.10

.30

.50

1.00

6.00

.10

.30

.15 .05

A. W. HUNT-(Christy) A beautiful glowing carmine red, extra large and very attractive. Gold stripe through center of petals

Large............

Medium........ .20

.55

1.00

2.00

12.00

Small 
BYRON L. SMITH-(K-1917). Re- ea.

fined lavender pink on white ground. Color equal to a choice catteyla orchid

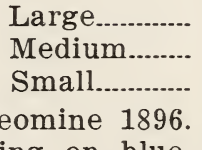
Dark violet bordering on blue, small flower but distinct shade, called a blue

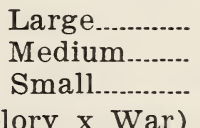

BEACON FLAME (Glory $x$ War) Self colored vermilion scarlet

Large.......-.
Medium.....-.
Small.........
1904). Lilac
ers open at

BERTREX-(Austin 1904). Lilac
white; many flowers open at one time

Large...........
Medium.......
Small..........
w e e 1910).

BALTIMORE - (C ow e e 1910).
Beautiful light salmon, deeper in throat

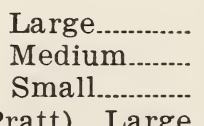
$.50 \quad 1.40$

$\begin{array}{rrrrr}.15 & .35 & .65 & 1.25 & 7.00 \\ .10 & .25 & .40 & .75 & 5.00 \\ .10 & .15 & .25 & .50 & 3.00\end{array}$

BENGAL TIGER-(Pratt). Large

blossoms of a peculiar shade of smoky red, striped and lined with smoky blue-grey. Barbarically gorgeous

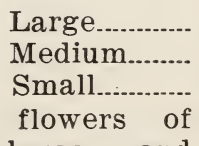

BERNICE - Large flowers of $\begin{array}{lll}.10 & .20 & .30\end{array}$ .60 striking reddish brown and orange

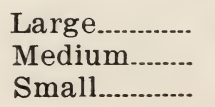


CANARY BIRD-(Prim) (Childs ea.

3

6

1910). Clear lemon yellow of good size and form

Large...........
Medium......
Small........-

CAPTAIN BOYNTON - (Boyn-
ton). Large, light lavender, with darker spots on lower petals-very fine Large............

Medium........

.15

.35

.65

1.25

.75

7.00

$.10 \quad .25$

.40

5.00

$.10 \quad .15$

.25

.50

3.00

Small

.25

.70

1.25

2.50

15.00

.40

.75

1.50

10.00

$.30 \quad .50$

1.00

6.00

CHICAGO WHITE - (K-1913).

White with lavender marks

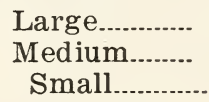

$\begin{array}{lll}.10 & .25 & .40\end{array}$

.75

5.00

$.10 \quad .15$

.25

.50

3.00

.25

1.50

CONSPICOUS - (Dames 1910).

Light blue, throat blotched yellow on lower petals. Unusually colored like a pansy

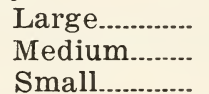

.25

.70

1.25

2.50

15.00

$.15 \quad .40$

.75

$\begin{array}{ll}1.50 & 10.00\end{array}$

$.10 \quad .30$

$.50, \quad 1.00$

6.00

CRACKERJACK-(Cowee 1903).

Dark brick red, small flower but very popular on account of its unique color

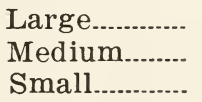

$\begin{array}{lll}.10 & .25 & .40 \\ 10 & .15 & .25\end{array}$

.75

5.00

$.50 \quad 3.00$

.25

1.50

CATHARINA-(Velthys 1916 ).

Light grayish blue; lower petals a little deeper in color with a brownish red spot. Tall spike and mighty pretty

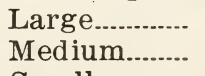

.12

.70

1.25

2.50

15.00

.40

.75

1.50

10.00

Small

.10

.30

.50

1.00

6.00

CARMEN SYLVA - (Prestgard 1917). Beautiful large snow white blossom on tall stems. Excellent cut flower

\begin{tabular}{|c|c|c|c|c|c|}
\hline Large.............. & .15 & .35 & .65 & 1.25 & 7.00 \\
\hline Medium......... & .10 & .25 & .40 & .75 & 5.00 \\
\hline Small...--_-.. & .10 & .15 & .25 & .50 & 3.00 \\
\hline
\end{tabular}


CHRIS - (Greuuemans 1919) ea.

$3 \quad 6$

Deep maroon red, $\mathrm{s}$ had ed purple

Large.

Medium........

Small

CRIMSON GLOW - (Betscher 1916) Rich, glowing crimson with velvety center. Large flower. A popular garden variety

Large...........
Medium.......
Small..........

.10

CHATEAU THIERRY - Bright .15 .10 .10
30. .20 .10
.75

.50

.30
1.50

1.00 .60
10.00 6.00 4.00

cerise with carmine spot on lower petals. Enormous wideopen flower 4 to 6 inches diameter. Fine spike, excellent grower. A sensation whever exhibited.

Large...........
Medium.......
Small........-
WONDER-

COWEES SCARLET WONDER-
(Groffs). Large, glowing, bril.20

.55 .15 .10
.50

1.00 .60 .30
6.00

4.00

2.00

liant, velvety, pure scarlet flowers. A very fine red

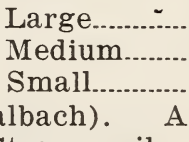

CLAREMONT - (Salbach). A
clear yellow self. Strong spike. .25

.70 .15 .10
6 to 8 open at one time.

Large.........-..

DAWN-Fine pink, large flowers on long spike

Large.

Medium........

Small.

.50

1.40

.80

.10

.30

2.50

1.50

5.00

3.00

1.00

30.00

18.00

.50

$.35 \quad 1.00$

1.75

1.00

.40
3.50

2.00

.75 .10 .25

DIANA-(Zeestraten). Gorgeous bright red blossoms on tall spikes. Wide open flowers well placed. An improvement over Crimson Glow.

Large. .15

Medium ........ $\quad .10$ Small 
DUCHESS OF YORK - (Dames). Dark blue, called an improved Baron Hulot

Large...........
Medium.......
Small.............

DAVID STARR JORDAN-(Die-
ner). Immense flowers of intense flame color with lighter speckled throat

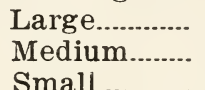

.25

.70

1.25

2.50

15.00

.15

.35

.65

1.25

8.00

.10

.25

.40

.75

5.00

DR. F. E. BENNETT-(Diener).

Flowers of largest size and most

fiery red imaginable. Called the

BEST RED

$\begin{array}{lrr}\text { Large............ } & 1.00 & 2.75 \\ \text { Medium....... } & .60 & 1.60 \\ \text { Small............ } & .40 & 1.10\end{array}$

5.00

3.00

10.00

60.00

2.00

6.00

36.00

4.00

24.00

DIENERS AMERICAN BEAUTY

-American Beauty rose color, creamy yellow throat-many open at one time-each spike a boquet in itself

Large.............
Medium........
Small............
-1918). Large

E. J. SHAYLOR-(K-1918). Large
ruffled blossoms, pure deep rose pink, almost coral red on good stem

Large.............
Medium........
Small............
IA_-(Velthys

EMPRESS OF INDIA-(Velthys
1918). Deep velvety maroon

(plum violet)

Large..............

Medium.......

Small.......... . .10

.10

.25

.40

.15

1.00

2.00

12.00

.65

1.25

8.00

.40

.75

5.00

.10

.25

.75

.50

5.00

3.00

$.25 \quad 1.50$

$15 \quad .40$

.30

.75

1.50

10.00

1.00

6.00

.20

.60

4.00

EUROPA-(Pfitzer 1911). Purest

white, lowest petals tinged cream

Large............ . .15

Medium........ . .10 
EVELYN KIRTLAND - (Austin ea.

1916). An exquisite shade of melting pink, deep towards the edge of the petals. Scarlet blotches on lower petals. Slender spike

Large.............
Medium........
Small.............
a n k 1922).

ELORA - (B u r b a n k 1922).
Creamy white, rich cream spots in throat on lower petals, spots overlaid with markings of pink

Large.............

Medium ........ . .15

.15

.35

.65

1.25

7.00

.10

.25

.40

5.00

.15

.75

3.00

EARLY SUNRISE - (Velthys Small...........
- Velthys
ering pinkish 1926). Large flowering pinkish
salmon with yellow throat. Very fine and meritorious

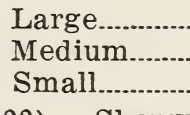

.25

.15

.10

.35

.70

.40

.30 .25
1.25

.75

2.50

1.50

1.00
15.00

10.00

6.00

E. G. HILL-(K-1922). Showy .10 .65 1.25 7.00 .75 5.00 .50 3.00 flower of finest salmon rose with large beautiful creamy throat

Large............. Medium.

$\begin{array}{lll}.50 & 1.40 \quad 2.50\end{array}$

$5.00 \quad 30.00$ $.80 \quad 1.50$

$3.00 \quad 18.00$

ENERGIE-Earliest bright scar.30 let

Large............
Medium.......
Small..........
- E a r l y.

.25

.70

1.25

2.50

15.00

.15

.75

1.50

10.00

.30

.50

1.00

6.00

ELIZABETH TABOR -
Pink with cherry blotch

Large............
Medium........
Small.........
17). Canary

FLORA-(Velthys 1917). Canary
yellow with slight markings in throat

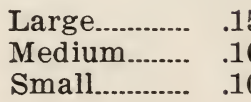


FAY LANPHIER-(Williams). A ea.

warm watermelon pink suffused coral, often having a crystaline texture on the petals as if frosted

Large.

.20

.55

Medium........

.15

Small

.10

.35

1.00

2.00

12.00

.25

.65

1.25

8.00

.40

.75

5.00

FERN KYLE-(K-1921). Ruffled, very large, creamy white with slight amaranth purple in throat

Large............

Medium........

Small

.35

1.00

1.75

3.50

20.00

.20

.55

.25

2.00

15.00

.10

.40

.75

5.00

GEORGE PAUL-(Lemoine 1893).

Harvard crimson

Large............

.15

Medium.

.10

.40

.75

1.50

10.00

Small.............

.10

.30

.50

1.00

6.00

GOLDEN WEST-(Wilmore 1907) .20

.30

.60 4.08

Rich nasturtium red with golden suffusion, lower petals tipped yellow

Large............

Medium........

Small

.10

.30

.30

.60

6.00

4.00

GRETCHEN ZANG - (Austin 1915). Large sparkling bloomsbeautiful soft pink, blending into scarlet on lower petals

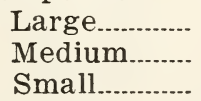

GLORY OF PEMBROOKE -

(Kir-choff). A tall spike, primrose yellow flowers

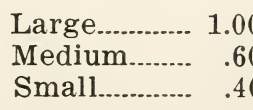

GLORY OF KENNERMERLAND

-A true rose colored glad, deep rose. Lower petals exhibit a pomegranite blotch on a creamy ground

Large.............

Medium........

Small 
GOLDEN MEASURE - (Kelway ea.

1912). Great tall upstanding spikes of pure golden yellow blossoms. Described as "rich as cream"

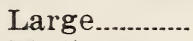

Medium..............

Small

.15

.10

.10
.40

.30

.20
.75

.50

.30
1.50

1.00

.60
10.00

6.00

4.00

GOLD DROP - (Prim) (K-1915). An extra large, pure deep yellow with red line on petals. Ruffled

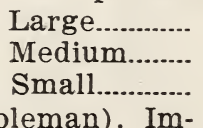

.15

.75

1.50

10.00

.10

.30

.50

1.00

6.00

.10

.20

.30

.60

4.00

GIANT NYMPH-(Coleman). Immense rose pink flowers with creamy yellow throats borne on tall erect stems. A wonderful glad.

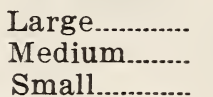

35

1.00

1.75

3.50

.20

.35

1.00

2.00

20.00

.15

.35

.65

1.25

15.00

GOLDEN KING-Clear, unfading

yellow with vivid crimson blotch in throat

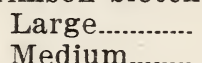

.15

.40

.75

1.50

1.00

10.00

.10

.50

Small

.30

.30

.60

6.00

.20

HELEN TODD-(Diener). Light
rose pink, deeply edged around each petal, throat deep scarlet

Large..........-

Medium........

Small

.10

.35

.65

1.50

.75

.25

.50

7.00

5.00

.15

3.00

HERADA-(Austin 1915). Only one of its color. Large blooms of clear mauve, small throat blotch of deep magenta

$$
\begin{aligned}
& \text { Large............ } \\
& \text { Medium........ } \\
& \text { Small............. }
\end{aligned}
$$

.25 .10 .15

.75

5.00

.50

3.00

25 .

1.50

HALLEY-(Velthys). Very large flowers of lovely salmon pink, lower petals blotched yellow with carmine flakings

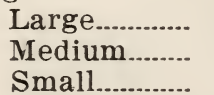


HENRY FORD-(Diener). Very

ea.

3

6

12

100

dark, rhodamine purple shading into deeper tones near the cen-

ter. An improved Anna Eberius

Large...........
Medium.......
Small..........
.25

.15

.70

.40

1.25

.75
$.30 \quad .50$

IDA VAN-(K-1915). Deep flaming cerise

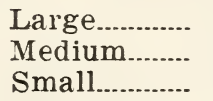

.15

.10

.10
.40

.30

.20
.75

.50

.30
2.50

1.50

1.00

15.00

10.00

6.00

INDEPENDENCE - (Woodruff).

An old favorite. Coral pink

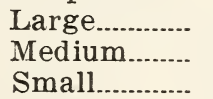

.40 .25
.75

.50

.25
1.50

1.00

.60
10.00

6.00

4.00

ILLUMINATOR - Brilliant carmine red, with faint suffusion of blue. An intense French purple blotch on lower petals

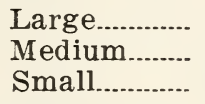

JACK LONDON-(Diener). Light salmon with brilliant orange flame stripes; golden yellow throat. Tall grower

$$
\begin{array}{ll}
\text { Large............ } & .15 \\
\text { Medium } & .10 \\
\text { Small_........... } & .10
\end{array}
$$

JOE COLEMAN - (K-1915). A beautiful ruffled flower of rich red. Strong and vigorous

$\begin{array}{lrrrrr}\text { Large........... } & .25 & .70 & 1.25 & 2.50 & 15.00 \\ \text { Medium...-... } & .15 & .40 & .75 & 1.50 & 10.00 \\ \text { Small......... } & .10 & .30 & .50 & 1.00 & 6.00\end{array}$

J. A. CARBONE-(Diener). Immense flowers of glowing orange salmon. Throat yellow. Tall erect stems

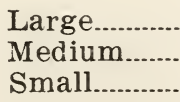


JEWEL - (Zeestraten) (Prim). ea.

3

6

Light salmon pink with golden

throat. Flowers large and wide open. Slender stem

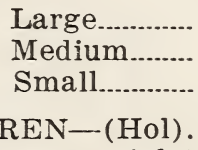

$.15 \quad .40$

.75

1.50

7.00

.10

.25

.40

.75

5.00

.10

.15

.25

.50

3.00

JACOBA VAN BEYEREN-(Hol).

Large flowers of pure violet on tall erect stems; well arranged and many open at one time

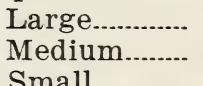

$\begin{array}{rrrrr}.25 & .70 & 1.25 & 2.50 & 15.00 \\ .15 & .40 & .75 & 1.50 & 10.00 \\ .10 & .30 & .50 & 1.00 & 6.00\end{array}$

KUNDRED'S GLORY - (K-1917).

The maternal ancestor of all the ruffles. Large ruffled blossoms of creamy apricot, tinted light pink

Large.............

Medium........

Small.

LAVENDER PRINCESS - (Williams). A lustrous combination of lavender and pink. Large flower

.20 $.55 \quad 1.00$

LIEBESFEUER-(Velthys 1912).

Very large flower of extremely dazzling coral spikes

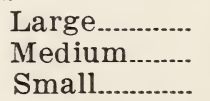

LE MARECHAL FOCH - (Van Duersen 1916). A beautiful shade of pink, flowers twice size of America

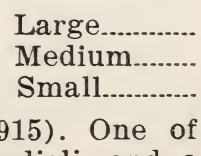

LILY WHITE-(K-1915). One of
the early white gladioli, and a florist's money maker

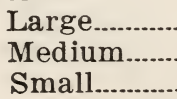


LOUISE-(Wright). A real beau- ea.

ty. Fine bright bluish lavender

with a wine colored blotch on

lower petals. Wide open flowers

Large............

.15

.35

.65

1.25

7.00

Small

.10

.40

5.00

$.25 \quad .25$

.75

3.00

LUCIE-A tall, growing prim of soft primrose yellow. Very large flowers

Large.............
Medium.......
Small............

L'IMMACULEE-Long spike of pure white blossoms, four open at one time. Very early

Large.............
Medium.......
Small...........
Houdyshell)

LOS ANGELES - (Houdyshell).
Immense spikes bearing huge blossoms of shrimp pink with throat of orange tinted carmine

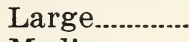

Medium.........

Small

MARY FENNELL - (K 1917).

Deep lilac, shading paler soft primrose yellow throat. Distinct, dainty and lovely

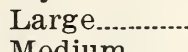

\section{Medium.....}

MYRTLE-(K 1915). Tender and delicate rose pink. Very early.

A beautiful thing.

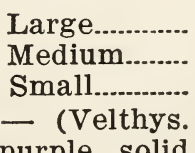

MASTER WIETZE - (Velthys.
1916). Pure royal purple, solid color

$\begin{array}{lrrrr}\text { Large............ } & .15 & .40 & .75 & 1.50 \\ \text { Medium....... } & .10 & .30 & .50 & 1.00 \\ \text { Small........... } & .10 & .20 & .30 & .60 \\ & & & & \\ & -15- & & & \end{array}$


MRS. FRANCES KING - (Cob- ea.

lentz 1909). Long spike of immense flowers of light scarlet or flame red, shaded salmon. An old variety, but extremely popular and fine Large............

Medium........ Small

.25

.40

.75

5.00

.10

.15

MRS. FRANK PENDLETON-(K 1915). Tall, majestic spikes of rose pink blossoms bearing a giant blotch of richest velvety red on lower petals. No finer variety-THE BIGGEST SELLER TODAY

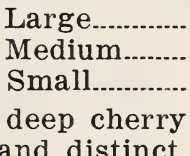

.25

.75

5.00

.10

.15

.40

.50

3.00

.25

1.50

MRS. WATT-A rich deep cherry red, very beautiful and distinct.

Large sized bulbs advisable

$$
\begin{aligned}
& \text { Large............. } \\
& \text { Medium -..-.... } \\
& \text { Small }
\end{aligned}
$$

MRS. DR. NORTON-( $\left.\begin{array}{ll}\mathrm{K} & 1915\end{array}\right)$

One of the most beautiful of glads. Silvery white blossoms daintily tinged pink, deepening towards the edges. Rich creamy throat. Resembles apple blossom tints

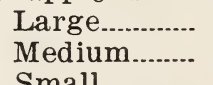

MURIEL - (Pfitzer). A lovely lavender variety. A orchid shade with purple blotch on lower petals

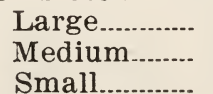

MAJESTIC-(Hopman 1916). An entirely new shade of beautiful orange salmon, slight white marks on lower petals

$$
\begin{aligned}
& \text { Large ............ } \\
& \text { Medium........ } \\
& .25 \\
& .70 \\
& 1.25
\end{aligned}
$$


MISS SPOKANE - $\left(\begin{array}{ll}K & 1922\end{array}\right)$, ea.

3

Large flowers of orange salmon, slightly deeper in throat. Six and seven open at one time

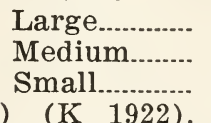

.20 .15 .10

MING TOY - (Prim) (K 1922).

.55

.35 .25

1.00
.65
.40

2.00

1.25 .75

Large blossoms of finest form well placed. Deep buff with a yellow throat

Large.............

Medium .........

Small

MARIE KUNDRED- (K 1918). Grandest of all whites-ruffled

$$
\text { Large........... }
$$

MR. TREUB-Apple blossom pink

Large............
Medium.......
Small..........

MAINE - (Vos). Light lemon, changing to pure white

Large..............

Medium.........

Small

MORNING GLORY-An improved

Schwaben. Color and markings a clear sulphur yellow with a small scarlet-red blotch on the lower petals. Very graceful spike. Arrangement of flowers like "Schwaben"

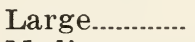

Medium........ .25

Small

MAIDEN'S B L U S H - (Prim).

Pink, mottled darker, lower petals have tiny crimson blotch on purpilish yellow ground. Very early forcer

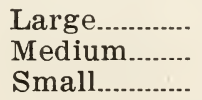


MYRA-(Prim) (N). New giant ea.

flowered Prim. Deep salmon over yellow ground, yellow throat, pink lines. Tall slender stem. Very showy

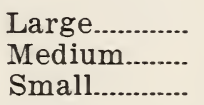

MRS. H. E. BOTHIN-(Diener)

(R). Flesh pink, tinted salmon, flame colored center. Heavily ruffled. Flowers large and correctly spaced on stem.

$$
\begin{aligned}
& \text { Large............. } \\
& \text { Medium........ } \\
& \text { Small.......... }
\end{aligned}
$$

.15

.10

.10

.40

.75

1.50

1.00

.60

10.00

.50

.20

.30

MRS. O. H. HALLIDAY-Delicate pink, cream blotch, long stem

$$
\begin{aligned}
& \text { Large............. } \\
& \text { Medium........ } \\
& \text { Small........... }
\end{aligned}
$$

.15

.10

.40

.30

.75

1.50

1.00

6.00

4.00

MONA LISA - $\left(\begin{array}{ll}K & 1917)\end{array}\right)$ ( $R$ ). .10 .20

.50

.30

Magnificent ruffled flowers of softest rose pink, almost a blush white. Extremely fine

$$
\begin{aligned}
& \text { Large............. } \\
& \text { Medium....... } \\
& \text { Small........... }
\end{aligned}
$$

MARSHALL FOCH-(K). Tall .10

spikes bearing ruffled flowers

of warm salmon pink, the salmon darker on the edge of the petals

$$
\begin{array}{ll}
\text { Large............. } & .20 \\
\text { Medium........ } & .15 \\
\text { Small........... } & .10
\end{array}
$$

See also LEMARECHAL FOCH for

Holland variety ( a pale pink).

MARY PICKFORD-(K). An extraordinary flower of delicate creamy white with throat of soft sulphur yellow. Even the stem and calyx are white

$$
\begin{array}{ll}
\text { Large............ } & .15 \\
\text { Medium........ } & .10 \\
\text { Small............ } & .10
\end{array}
$$


MR. MARK-(Velthys 1916). A ea. pleasing shade of light blue with darked blotch

Large.............
Medium........
Small...........

MRS. F. C. PETERS-(Fischer). .20 .15 .10 .55 .35 .25

Fine rosy lilac blossoms with soft crimson blotch; slightly ruffled and many opened at one time. Tall, vigorous and fine

$$
\text { Large............ }
$$

$$
\text { Small. }
$$

.55

.35

.25

MR. W. H. PHIPPS-(Diener). .10

LaFrance pink, tinged light rosy salmon; lower petals striped and speckled red. Enormous flowers on long stately spikes

$$
\begin{aligned}
& \text { Large............. } \\
& \text { Medium........ } \\
& \text { Small............ }
\end{aligned}
$$

.75

.50

.30
2.00

1.35 .80
3.75

2.50

1.50
7.50

5.00

3.00
50.00 32.00 20.00

MRS. W. E. FRYER-(K 1915). Large cheerful flowers of brilliant orange scarlet on strong stems

Large.............

Medium........

.35

Small .10

MRS. LEON DOUGLAS-(Diener). Enormous rose colored blossoms striped with scarlet. Extra long spikes

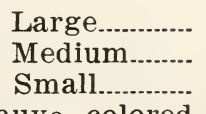

MRS. HARDING-Mauve colored flower with feather markings

$$
\text { Large.......... }
$$

favorite hyacinth flowered. Delicate cream blending to canary yellow, throat splashed crimson

$$
\begin{array}{lrrrrr}
\text { Large........... } & .10 & .30 & .50 & 1.00 & 6.00 \\
\text { Medium....... } & .10 & .20 & .30 & .60 & 4.00 \\
\text { Smali.......... } & .05 & .10 & .15 & .30 & 2.00 \\
& & & & &
\end{array}
$$


NORA-(Velthys 1916). Beautiful ea.

light lavender blue with a dark er spot on lower petals. One of the best orchid shades
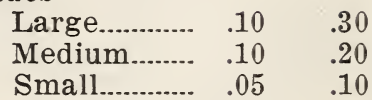

.50

1.00

6.00 .10

.20

.30

.60

4.00

.05

.10

.15

.30

2.00

NOXALL - ( K 1918). Massive, pure white with dainty beautiful throat

Large.

.50

2.50

5.00

30.00

Medium........

.80

1.50

3.00

18.00

Small

.20

.55

1.00

2.00

12.00

ODIN-Dark salmon pink with bright carmine blotch. A beauty

Large............

Medium........

Small

.15

.10

.40

.75

1.50

10.00

.30

.50

1.00

6.00

.10

.20

.30

.60

4.00

ORANGE GLORY-(K 1915) (R). Gleaming, ruffled, orange colored blossoms with lighter throat. Robust grower

Large.

Medium

Small............

.15

.40

.75

1.50

10.00

.10

.50

1.00

6.00

.10

.20

.30

.60

4.00

ORANGE FLAME-(K 1922) (R). A wondrous glowing vermilion orange. Very large blooms, heavily ruffled

Large............

Medium........

Small.............

.50

1.40

2.50

5.00

30.00

$.80 \quad 1.50$

3.00

18.00

$.20 \quad .55 \quad 1.00$

2.00

12.00

ORANGE QUEEN-(Prim). An extraordinary and outstanding prim of warm apricot

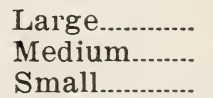

1.25

2.50

15.00

.40

.75

1.50

10.00

.10

.30 .50

1.00

6.00

ORANGE PRINCE-(Vos). One of those rare colors among the Prims. Capucine orange slightly tinted deeper orange. Tall straight spikes with many flowers open at one time

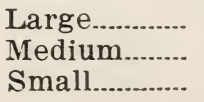


PARAMOUNT-(K 1918). A won- ea. derful blending of pink and cream. Similar to Mrs. Dr. Norton

Large.................

Medium.........

Small

PURPLE GLORY-(K 1926) (R). Deepest velvety maroon-red with almost black blotches. A perfect giant among gladioli. Recommended

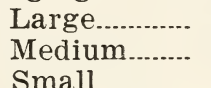

.25

.15

.10

PROF. A. C. BEAL-(Kirchoff). Deep maroon, a flower of good merit

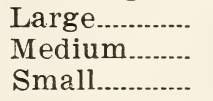

.50

.30

.20

PINK WONDER-LaFrance pink with yellow shading at base of lower petals, overlaid with a feathering of rose. Extra good pink

Large...............

Medium.........

Small

.1

PYTHIA-(K 1918). Magnificent stately spikes of gigantic red blossoms, several open at one time. Showy in the extreme

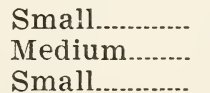
.20

.55

1.00

.15

.10

.35

.25

A fine

PANAMA - (Banning). deep lavender pink, a true rose color. Fine for bedding

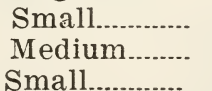

.05

.20

.30

.60

.40

.20
12.00

8.00 5.00

PEACE-(Groff 1918). Extra tall spikes of pure white blossoms with lilac feathering in lower petals

Large...............

Medium

.10

.20

.05

12

Small 
PRINCE OF WALES-(VanZant- ea.

3

6

en 1910). Softest salmon with an apricot undertone. Large flowers and very early. One of the biggest sellers

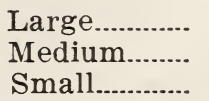

PRINCE OF WALES IMPROVED -Dark salmon pink with a bright carmine blotch. A beauty, somewhat resembling a seashell

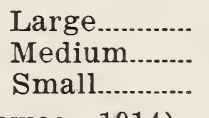

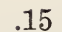

.10

.10

.40

.30

.75

.50

1.50
1.00

.20
10.00

6.00

.60

PEACH BLOW-(Cowee 1914). An old fashioned favorite with deep pink, but small flowers

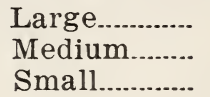

$\begin{array}{lllll}.10 & .20 & .30 & .60 & 4.00 \\ .05 & .12 & .20 & .40 & 2.40 \\ & & & .20 & 1.00\end{array}$

PURITY-(Pommert). A pure white with flowers and habit like America. Very good substance

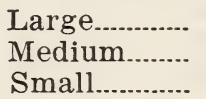
.10

PRIMROSE BEAUTY-(K 1918). (Prim). A very pretty yellow prim. Tall, strong spikes of large primrose yellow. Early

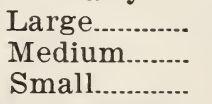
.15 .40 .75

PINK PERFECTION- (Hopman 1913). An exquisitely lovely flower of La France pink; throat paler with a peculiar frosted appearance. Graceful willowly stems, and many open at one time

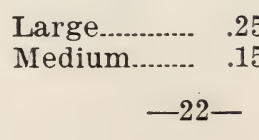


PRINCEPS - (VanFleet 1897). ea.

3

6

Rich dark scarlet marked with

white, foliage dark green

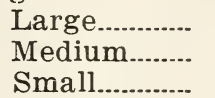

PAR EXEL-A round petaled salmon queen

Large............

Medium........

.15

Small

.10

.40

.75

1.50

10.00

.30

.50

1.00

6.00

.10

$.20 \quad .30$

.60

QUEEN OF THE BLUES-Light pinkish lavender. Small bur gundy blotch on yellow ground on lower petals. Tall spikes well arranged

Large.............

Medium........

Small

$\begin{array}{lll}.25 & .70 & 1.25\end{array}$

2.50

1.50

15.00

.15

.40

.75

1.00

10.00

$\begin{array}{lll}.10 & .30 \quad .50\end{array}$

6.00

RED EMPEROR - (Vos). Extremely large flower of good scarlet red

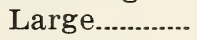

Medium..........

.25

.15

.70

.40

1.25

2.50

1.50

15.00

.10

30

.75

1.00

10.00

6.00

ROSELLA-Deep magenta shaded cerise, and shaded to white base with blotch of deep crimson

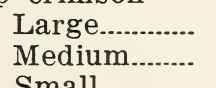

.15

.40

.75

1.50
1.00

10.00

.10

.50

6.00

Small

.10

20

.60

4.00

RADIANT MORN - Beautiful deep pink

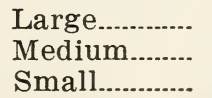

.15

.10

.40

.30

.75

1.50

10.00

.50

1.00

6.00

$.10 \quad .20$

.30

.60

4.00

ROSE GLORY-(K 1916) (R). A very large and splendid ruffled flower of purest pink; deep blotch in the throat

Large...........
Medium........
Small...........
lor of Pink

ROSE PRECOSE-Color of Pink Perfection

Large............

Medium........ .15

.35

.65

1.25

.75

7.00

.10

.40

5.00

$.10 \quad .15$

.25

.50

3.00

Small:

.25

.70

1.25

2.50

1.50

15.00

.40

.75

1.00

10.00

.10

.30

.50

6.00 
RADIUM-LaFrance pink tinted ea.

lighter. Lower petals have a scarlet blotch on a creamy white ground

Large.............
Medium.......
Small..........
rower terms

RED CANNA-One grower terms
it the "tallest glad" having grown it six feet in height.

Brilliant self color

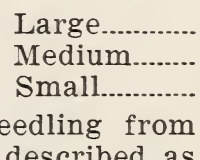

.15
.10
.10

.40
.20
.20

.75

$1.50 \quad 10.00$

.50

1.00

.60

Large.............
Medium.......
Small.........
edling from aWilbrink without the yellow marking on the lower petals. Flowers are large, well expanded with a pearl-like lustre

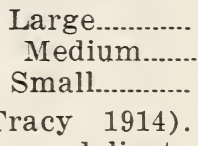

.25
.15
.10

1.25

2.50

15.00

.75

1.50

10.00

$.30 \quad .50$

1.00

6.00

ROSE PEARL-A seedling from
Wilbrink. Can be described as An old favorite, a delicate cream tinted with pink; lower petals have torch-like tongue

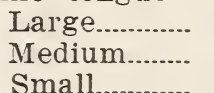

$\begin{array}{rrrrr}.10 & .30 & .50 & 1.00 & 6.00 \\ .10 & .20 & .30 & .60 & 4.00 \\ .05 & .10 & .15 & .30 & 2.00\end{array}$

1910 ROSE - (K 1915). Very

$\begin{array}{cccrr}.15 & .40 & .75 & 1.50 & 10.00 \\ .10 & .30 & .50 & 1.00 & 6.00 \\ .10 & .20 & .30 & .60 & 4.00\end{array}$

large flower of pure, clear rosepink; white central lines on lower petals. Very good sub stance and good keeper

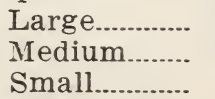

.25

.75

5.00

.10

.40

.50

3.00

.25

1.50

ROBERT J. KUNDRED - (K 1918). Medium tall, very brilliant orange vermilion. Very showy self color

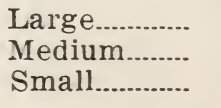

$.35 \quad 1.00$ 
ROSE ASH-(Diener). The color

ea. 3

36

an exquisite old rose, blended with ashes of roses; a lovely combination of color, fine ruffled, a tall and straight grower

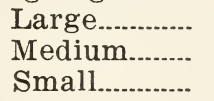

ROANOKE-(Prim). Rich, yellow prim. Vigorous and strong

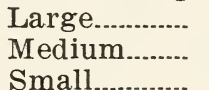

RED AMERILLAS-(K). Bright blue-red flower of giant size on medium height stems. Most gorgeous and showy red

$\begin{array}{lrrrrr}\text { Large........... } & .15 & .35 & .65 & 1.25 & 10.00 \\ \text { Medium....... } & .10 & .30 & .50 & 1.00 & 6.00 \\ \text { Small............ } & .10 & .20 & .30 & .60 & 4.00\end{array}$

REMEMBRANCE-Deep salmon p i n k blossoms. Prominent throat blotches. Tall spikes with many ruffled flowers open at one time

Large...........
Medium........
Small.........
seedling of
wer of rich. Princeps. Entire flower of richest pure colored self red. Very showy. Another finest red

$\begin{array}{lrrrrr}\text { Large........... } & .15 & .35 & .65 & 1.25 & 10.00 \\ \text { Medium....... } & .10 & .30 & .50 & 1.00 & 6.00 \\ \text { Small............ } & .10 & .20 & .30 & .60 & 4.00\end{array}$

ROOSEVELT - Described as a grotesque flower of reddish, yellow and pinkish colorings, all blended together. A very popular variety in our show grounds

$\begin{array}{lrrrrr}\text { Large........... } & .25 & .70 & 1.25 & 2.50 & 15.00 \\ \text { Medium....... } & .15 & .40 & .75 & 1.50 & 10.00 \\ \text { Small............ } & .10 & .30 & .50 & 1.00 & 6.00 \\ & & & & & \end{array}$


SCHWABEN - (Pfitzer 1913). ea.

Large heavy amber yellow with small red blotch deep in throat

Large.............

Medium.

.10

.25

.40

.75

5.00

Small

.10 .

.15

.25

.50

3.00

.25

1.50

SULPHUR KING - (Westerbeek 1918). Sulphur yellow, small flower

Large............

Medium........

Small

.20

.55

1.00

2.00

12.00

.15

.35

.65

1.25

8.00

.10

.40

.75

5.00

SUMMER-Mauve lilac similar to Herada but with throat flaked

Large...........

Medium.........

Small.........

.15

.40

.75

1.50

10.00

.10

.30

.50

1.00

6.00

.10

.20

.30

.60

4.00

SIR ROGER CASEMENT-(Diener). Dark maroon-heavily ruffled

Large............

Small.

SCARLET PRINCEPS--(K 1917).

Six or more massive Princeps flowers (scarlet) open at one time. Throat a little deeper. On the Coast called VIRGINIA

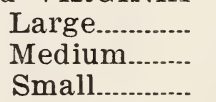

$\begin{array}{rrrrr}.10 & .30 & .50 & 1.00 & 6.00 \\ .10 & .20 & .30 & .60 & 4.00 \\ .05 & .10 & .15 & .30 & 2.00\end{array}$

SHEILA - (Coleman). Gorgeous salmon, extremely early. Called by some an improved Prince of Wales

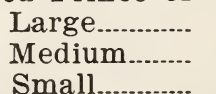

.35

.20

1.00

.55

.15

.35

1.75

1.00

.65

3.50

2.00

20.00

15.00

1.25

8.00

.40

.75

1.50
1.00

10.00

$\begin{array}{lll}.10 & .30 & .50\end{array}$

.30

.60

6.00

.10

.20

SWEET LAVENDER - Coleman). A lovely combination of light lavender pink blending into creamy yellow, with a prominent magenta blotch

$\begin{array}{lrrrrr}\text { Large............. } & .15 & .40 & .75 & 1.50 & 10.00 \\ \text { Medium........ } & .10 & .30 & .50 & 1.00 & 6.00 \\ \text { Small_.......... } & .10 & .20 & .30 & .60 & 4.00\end{array}$

SALMON QUEEN-See Baltimore. 
SALMON BEAUTY-(K 1915). ea.

(Prim). Tall graceful spikes of large showy salmon flowers with throat of pure yellow

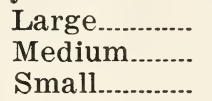
.15

SNOW GLORY - K 1917). Purest all white with only slight clear iris blue lines on lower petals

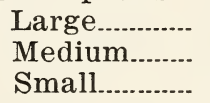

SOUVENIR - (Prim). Extremely clear yellow prim, long spike with many open at one time. The best cheap yellow

\begin{tabular}{|c|c|c|c|c|}
\hline Large............... & .15 & .35 & .65 & 1.25 \\
\hline Medium......... & .10 & .25 & .40 & .75 \\
\hline Small.............. & .10 & .15 & .25 & .50 \\
\hline
\end{tabular}

SCARLANO-(K). Bright orange red finely ruffled, extremely bright spot in the garden

\begin{tabular}{|c|c|c|c|c|c|}
\hline Large............... & .15 & .40 & .75 & 1.50 & 10.00 \\
\hline Medium......... & .10 & .30 & .50 & 1.00 & $6.0 \mathrm{c}$ \\
\hline Small_............ & .10 & .20 & .30 & .60 & 4.00 \\
\hline
\end{tabular}

SCARLET WONDER - (Groff).

Unquestionably the best red. Color, brilliant, glowing crimson-scarlet, self color, practically without streaks or marks

$$
\begin{aligned}
& \text { Large............ } \\
& \text { Medium....... } \\
& \text { Small........... }
\end{aligned}
$$$$
.25
$$$$
.70
$$

THE PEARL-(Westerbeck 1918). Delicate light pink with a creamy throat; several flowers opened at one time; slender stems 
TROTH-(Prim). White Prim ea.

variety with light cream in center

Large.............

Medium..

Small.

TOPAZ-(Prim). Unusually colored Prim hybrid;salmon pink tinged with buff, long stems

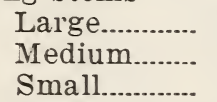

.10

.25

.15

.40

.75

.50

.25

7.50

5.00

50.00

32.00

3.00

20.00 5.00 3.00 1.50

THOS. T. KENT-(Diener). Very attractive flower combination of strawberry and shell pink

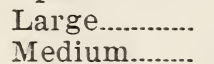

Small............. .25 .15

.70 .40

1.25

2.50

15.00 $.10 \quad .30$ .75 1.50 10.00 1.00

THEDA-(Bill). Most delicate shrimp pink gradually blending to soft Pinard yellow, with pansy lines on flower petals. Early

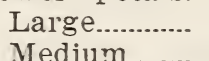

.15

.40

.75

1.50

10.00

.10

.50

Small

.10

.30

1.00

6.00

.20 .60

4.00

UHLAN CHIEF-(K). Flowers of most intense brilliant vermillion scarlet; lower petals deeper color and beautiful

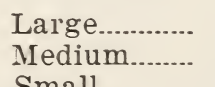

$.35 \quad 1.00$

1.75

3.50

20.00

.20

.55

1.00

2.00

15.00

Small

$.15 \quad .35$

.65

1.25

8.00

VIRGINIA - (Pooley). Identical with Kundreds Scarlet Princeps. California's greatest sensation red gladioli

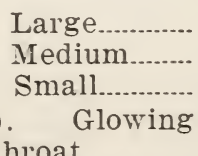

.30

.50

1.00

6.00

$.10 \quad .20$

.30

.60

.10

.30

4.00

.05

.15

2.00

VIRGINIA - (Tully). Glowing purple with white throat

$$
\begin{aligned}
& \text { Large........... } \\
& \text { Medium....... } \\
& \text { Small........... } \\
& \text { ). As the }
\end{aligned}
$$

VIOLET - (Kirchhoff). As the 
WAR - (Groff). Erect, stately spikes of blood-red flowers.

$\begin{array}{llrrrrr}\text { Late flowering } & \text { Large............. } & .10 & .30 & .50 & 1.00 & 6.00 \\ & \text { Medium...... } & .10 & .20 & .30 & .60 & 4.00 \\ & \text { Small_........... } & .05 & .10 & .15 & .30 & 2.00\end{array}$

WHITE GLORY-(K 1915) (R).

Pure white with an iris blue throat. Intensely ruffled

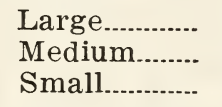

$\begin{array}{rrrrr}.20 & .55 & 1.00 & 2.00 & 12.00 \\ .15 & .35 & .65 & 1.25 & 8.00 \\ .10 & .25 & .40 & .75 & 5.00\end{array}$

WILBRINK - (Hopman 1916).

Flesh pink, creamy blotch in lower petals

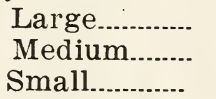

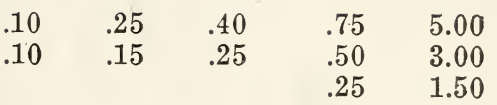

\section{GLADIOLI IN MIXTURES}

Mixtures outside of the Primulinum Hybrids are "built to order." You may in order ask for absence of any color. Each mixture is made for the occasion, with equal number of each color. All named varieties. We do not plan to put PRIMS in regular mixtures.

MIXED GLADIOLI-Department store quality. Contains not less that 10 varieties. Full range of color, all strong growers, large flowering sorts.

Large size. dozen. $50 \mathrm{c}$

Medium size....dozen.......................25c

100 for $\$ 3.00$ 100 for 1.50

PRIMULINUM HYBRIDS-All pastel shades. Well balanced strain rieties of gladioli. Contains many varieties not included in Departmen Store Mix.

Large size dozen. $.75 \mathrm{c}$

Medium size....dozen $40 \mathrm{c}$

100 for

100 for

PRIMULINUM HYBRIDS-All pastel shades. Well bananced strain of these wonderful tinted glads. (See next sheet).

Large size........dozen.. $50 \mathrm{c}$

100 for $\$ 3.00$

Medium size....dozen. $25 \mathrm{c}$

100 for. 1.50

WHY NOT place your order for delivery on 500 or 1000 Mixed Gladioli to be delivered to you EACH MONTH the balance of the planting season? 


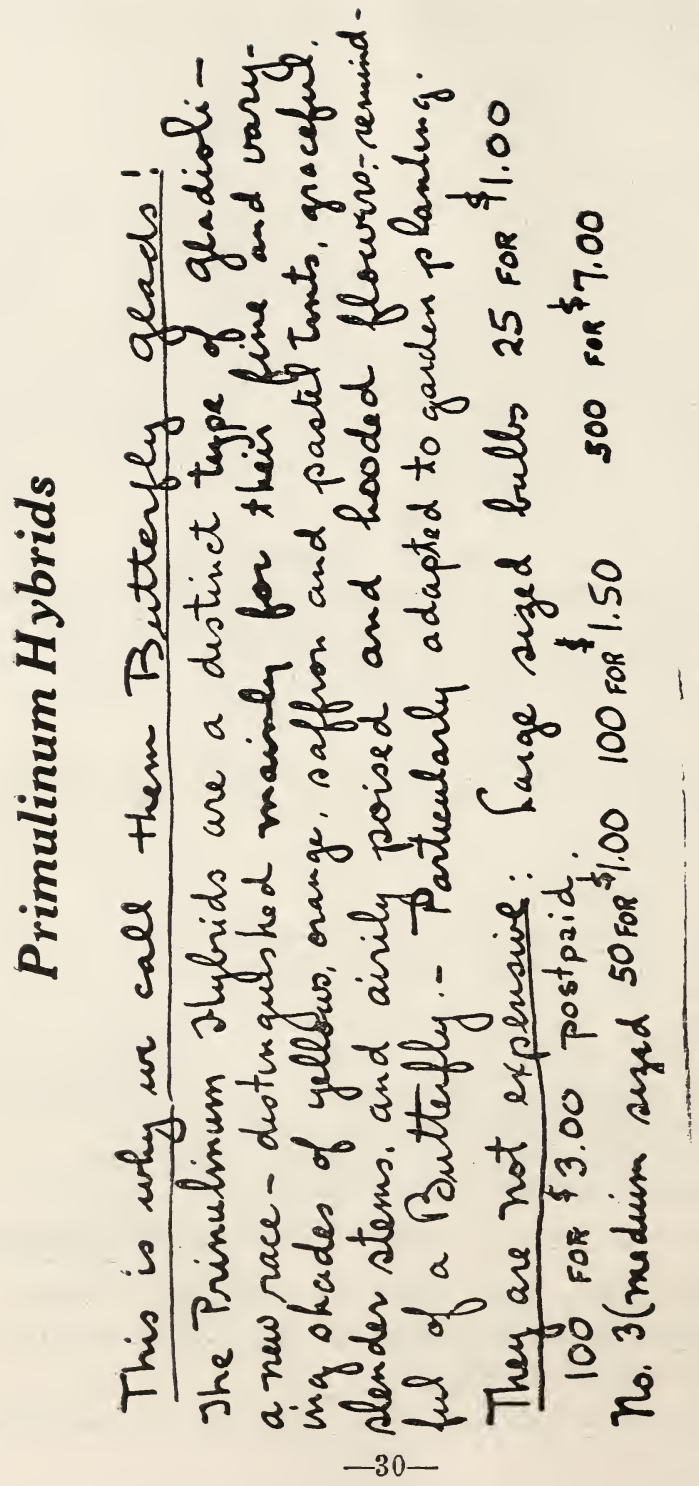




\section{Gift Boxes for the Garden}

Make very attractive and lasting remembrances for your friends or the amateur gardener. Such a gift gives great pleasure, as the recipient has the pleasure of planting and growing these flowers.

To meet an increasing demand, we are packing some of the varieties of bulbs in cartons suitable for gift boxes, two of which are described here. We can make up these boxes in any special mixture or price.

\section{Garden Bulbs}

Containing our 50 seasonable bulbs, including gladioli, daffodils, hyacinths and tulips. $\$ 3.00$.

These boxes are also packed with other bulbs and in all sizes, as listed under the various varieties.

\section{"To Make the New Year Glad Just Send a Box of Glads"}

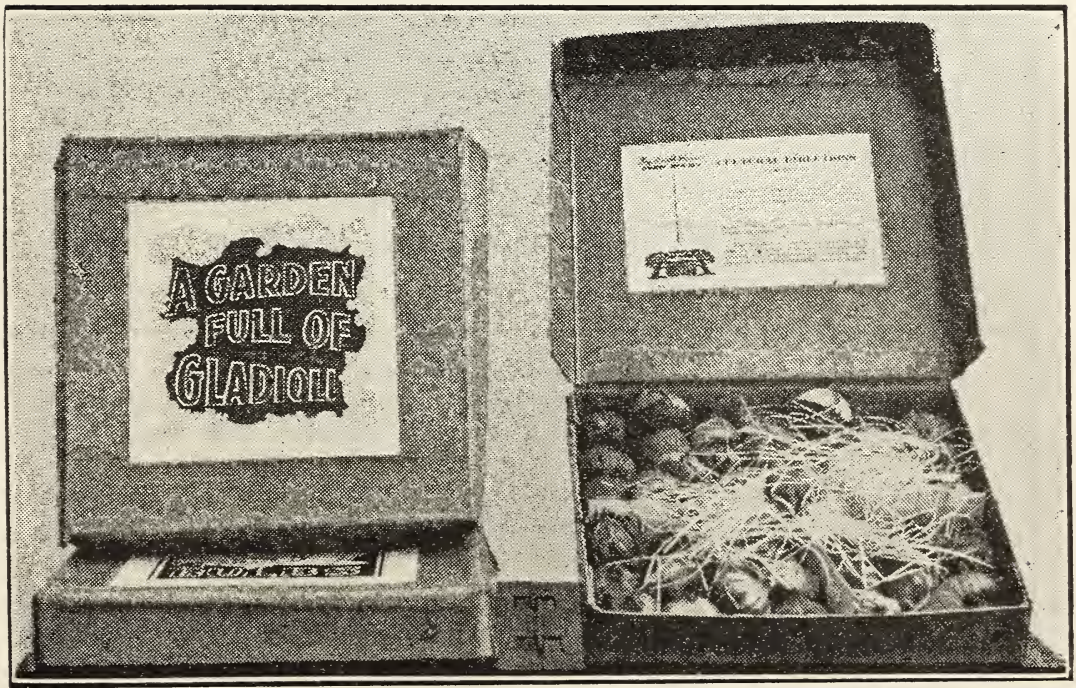




\section{GLADIOLI SEED}

Many of the most wonderful commercial sorts of gladioli in demand today were chance hybrids. A wonderful opportunity for you to "produce" a new gladioli. The seed can be grown to produce flowers the first year if sown early and will make splendid bulbs for the second year blooming.

SHOW GARDEN MIXED-Seed taken from seed pods from our trial show garden-all colors.

PRIMULINUM HYBRIDS-Flowers of distinct form which possess the clearest tone and tints of color to be found. From a very fine balanced color assortment.
100 seeds.........25c
1000 seeds.
$\$ 1.75$
COLLECTIONS OF GLADIOLI BULBS

\section{GARDEN FULL OF GLADIOLI}

A box of 50 bulbs, representing every color of the gladioli kingdom. All large sized blooming bulbs, in a special packed box ready for shipping $\$ 3.00$

HALF GARDEN-Same as the above, but containing

25 bulbs in mixture. $\$ 1.50$

\section{"ONLY-ONE" COLLECTION}

This has been entirely revamped this season. JUST THE THING FOR THE BEGINNER. One each of twenty five varieties of standard named varieties of gladioli, well balanced in color, large blooming bulbs. An exceptionally good value, sold for educational purposes. Never before offered at this price. Bulbs are NOT labeled as to name.............\$1.50

\section{"THE BANKER'S 200"}

In filling an order for 200 mixed gladioli for an Oakland banker, we thought of adding a little service and kept the varieties separate and so labeled them. The order has brought in so many return or repeat orders, that we include it now as a regular collection. All large sized blooming bulbs, ( $1 \frac{1}{4}$ inch and $1 \frac{1}{2}$ inch and up), 20 each of 10 varieties, named on the bags, our choice of varieties but full range of color, or you may suggest color preference. A wonderful value 


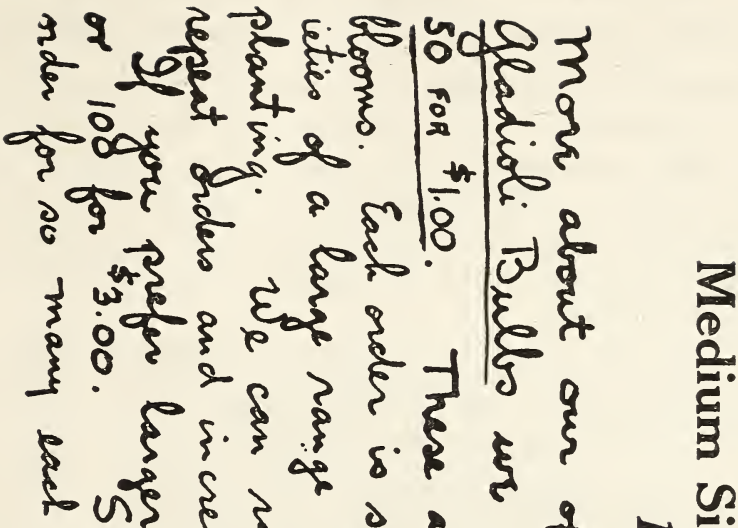

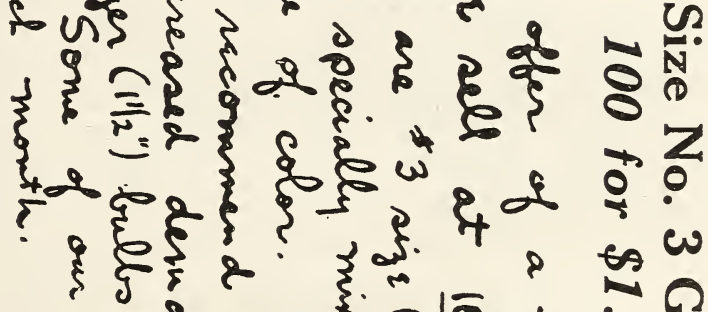

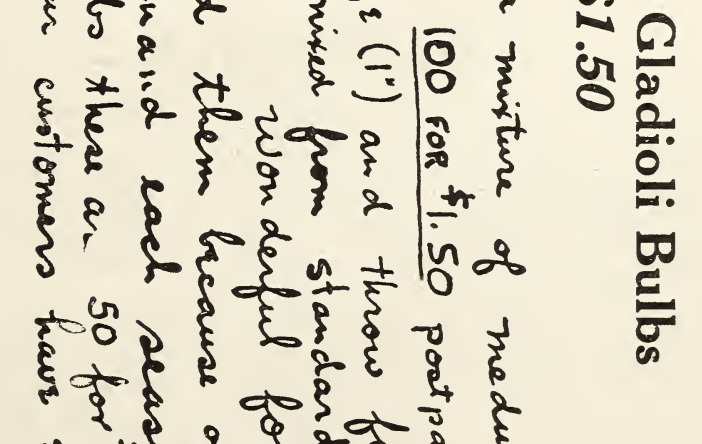

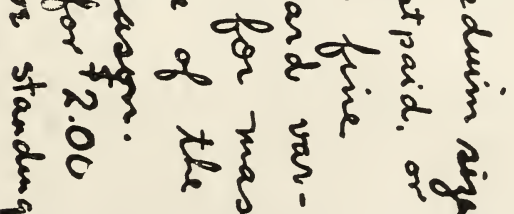

$$
\begin{aligned}
& \rightarrow \text { र है }
\end{aligned}
$$




\section{MINIATURE GLADIOLI}

\section{Plant October-November}

Blossom Time May-June

The culture of these Miniature Gladioli is very easy. Plant from October to December and you will be rewarded with wonderful blooms in May and June. Plant the bulbs 6 inches apart and 3 to 4 inches deep. Mass planting is very effective, and in our own growing we often get three or more spikes from one bulb. The flowers are borne on long slender stems and are in great demand as cut flowers.

APOLLO-Salmon with darker throat........ $\$$
ACKERMANI-Salmon pink, carmine throat
BLUSHING BRIDE-Ivory with crimson

throat

Each Doz.

$\$ .10$

BRILLIANT-The brililant scarlet.

.10

$\$ .75$

.75

100

CRIMSON QUEEN-Deep crimson

.10

.10

.10

PEACH BLOSSOM-Most popular rosy pink .10

RUBRUM (Red Bride)-Dark red inside

with green back.

THE BRIDE-Pure white

.10

WILLIAM III-Terra cotta.

.10

MIXED BABY GLADIOLI, good assortment

\section{DAFFODILS}

Plant October-December-Flowers February-March

To briefly explain the words Narcissus and Daffodil-Daffodil is the English name for a large family of plants and Narcissus is the Latin name; in other words, they are the same thing. However, custom has decreed that they do not mean the same thing, hence the confusion.

The word Narcissus is generally confined to the pure white petaled division like the Poeticus and to the bunch flowered varieties like the paper white. All others are in popular language Daffodils.

We list only the better and the newer varieties. They bloom in March. For outdoor planting, set before December in rich ground four inches below the surface and four to six inches apart.

Note: Due to existing state and federal regulations covering the shipment of narcissus and daffodils, all orders are accepted subject to securing stock meeting these many restrictions. 
PAPER WHITE GRANDIFLORA-Pure white.

Ea. doz. 100

May be grown in pebble bowl.

Large. $\$ .10$

$\$ 1.00$

$\$ 6.00$

Medium.

.10

.50

4.50

GRAND SOLEIL D'OR-Yellow, with orange cup

.15

1.25

9.00

GRAND MONARQUE-White, with orange cup

.10

1.00

6.00

GRAND PRIMO-White, with citron cup............

.10

1.00

5.00

THE PEARL-Entirely white.

1.00

6.00

MIXED POLYANTHUS-

POETICUS AND POETAZ TYPES (Parvi-Coronati)

POETICUS HORACE-Pure white perianth, rich solid red-eye, free flowering.

.15

1.25

9.00

POETICUS KING EDWARD-(Almira). Broad pure white perianth, orange scarlet crown.... POETICUS ORNATUS-Pure white perianth, broad cup margined scarlet.

.15

1.25

9.00

10

1.00

6.00

POETAZ ADMIRATION-Pale yellow, cup yellow edged, very fine.

.15

1.25

9.00

POETAZ KLONDYKE-Yellow with deep yel-

low eye

.15

1.25

9.00

\section{DAFFODILS}

AJAX TRESERVE-Light yellow, large trumpet BARRI CONSPICOUS-Broad yellow perianth, broad short cup, edged bright orange scarlet, very free bloomer, tall.

.75

8.00 60.00 INCOMPARABLE GLORIA MUNDY - Clear yellow perianth, much expanded; orange scarlet cup

INCOMPARABLE SIR WATKIN-Sulphur petals, deep yellow cup.

.15

1.25

9.00

.15

1.25

9.00

BI-COLOR VAN WAVERENS GIANT-Perianth primrose with bright yellow trumpet.... KING ALFRED-Golden yellow perianth and trumpet, the latter of beautiful form with deeply frilled mouth, large bold flower............. Small (flowering) size.

OLYMPIA-The trumpet is yellow and the perianth slightly lighter. 


\section{CROCUS}

(PLANT OCTOBER-DECEMBER)

(FLOWER IN EARLY SPRING)

Crocus are of very easy culture. Beautiful upright bell-shaped flowers, with grass-like foliage, and very early. They are at home if planted in grass plots or lawns and make fine borders and potted plants.

Doz.

100

Blue and purple shades $\$ .25$

$\$ 1.6 !$

White shades.

Yellow varieties

.25

1.60

Striped varieties.

.25

Mixture of above four

.25

\section{HYACINTHS}

\section{PLANT OCTOBER-DECEMBER-FLOWER IN MARCH}

Hyacinths are very fragrant and come in a great variety of colors. They make a magnificent display when grown outside in a bed. They are also very satisfactory. when grown in pots or in glasses of water. We are listing the single type only, as they are far superior to the double, and more certain to bloom. For outdoor culture set the bulbs so that the tops will be 3 to 4 inches below the surface and 6 to 10 inches apart. For pot culture use a 4-inch pot for a bulb or a large pot for 2 or 3 bulbs, and leave the tops of the bulbs just above the surface. After potting give a good watering and set away in a cool place and cover with coal ashes or sand until thoroughly rooted, which takes from $\&$ to 10 weeks. During this time do not allow them to become dry. They may then be set in a cool, light place until the flower spikes have developed, when they may be placed wherever they are wanted.

Due to the exceedingly high prices on hyacinths we omit the named varieties, advising our patrons to use the bedding size.

\section{BEDDING HYACINTHS}

These are good strong bulbs, imported direct from Holland, and will produce fine flowers. Exceptionally fine for bedding in mass effect.

\section{MIXED SHADES}

Bedding size, $14-16 \mathrm{~cm} ., 15 \mathrm{c}$ each, $\$ 1.50$ per 12

Miniature size, $12-14 \mathrm{~cm} ., 10 \mathrm{c}$ each, $\$ 1.20$ per 12

Light blue shades, mixed. Rose and pink shades, mixed.

Dark blue shades, mixed. White shades, mixed.

Dark red shades, mixed. Yellow shades, mixed.

All colors, mixed. 


\section{SCILLAS}

(Squills or Woods Hyacinths)

As edgings and masses in beds and borders, Scillas are most valuable. They succeed in shady flower borders and bare shady places and odd corners which often have a desert-like appearance in the flower garden can by their use be rendered bright and cheerful. Prices per dozen and 100 include postage.

SIBIRICA-(The Blue Squill). Bright blue, nothing more beautiful

for edgings or pots in eary spring, flowering with the Snowdrop.

Height, 4 inches. Mammoth bulbs. Doz., 80c; 100, $\$ 5.50 ; 1000$, $\$ 50.00$.

CAMPANULATA-Clear blue, very erect in habit (15 inches). Doz.,

$75 \mathrm{c} ; 100, \$ 5.00 ; 1000, \$ 45.00$.

Alba-Pure white (12 inches). Doz. 75c; $100, \$ 5.00 ; 1000, \$ 45.00$.

Rosea-Fine rose (15 inches). Doz. 80c; 100, $\$ 5.50 ; 1000, \$ 50.00$. PERUVIANA COERULEO-(The Cuba Lily) (Peruvian Hyacinth)

Very attractive in the herbaceous border or bed. Height 6 to 12 inches. Broad conical compact raceme of (100-150) purplish flowers. 35c each; $\$ 3.50$ per dozen.

\section{TULIPS \\ DARWIN TULIPS \\ PLANT OCTOBER-DECEMBER-FLOWER IN APRIL}

Darwin Tulips are beautifully outlined, cup-shaped and carried on tall, stiff stems. They vary in color from almost jet black, crimson and scarlet, purple to lilac, mauve, pink and indescribable intermediate blending and pastel shades. Yellow is the one color missing, but this can be supplied from the Cottage varieties listed on following pages and from the richly blended Breeders, varieties may be chosen which will increase the effectiveness of a planting of the pastel tints so prevalent among the Darwins.

PLANTING. Plant four or five inches deep and six inches apart in raised beds or border's so that the bulbs are slightly above the level of adjacent lawn or walks. In preparing the ground, use Bone Meal (three handfulls to a square yard), thoroughly mixed throught the soil.

BARONNE DE IAA TONNAYE-Bright rose, margined lighter

Ea.

Doz.

100 $\$ .10$

CLARA BUTT-Soft salmon rose.

DREAM - Beautiful lilac

GLOW-Brilliant vermilion, white and blue base 
GRETCHEN - Soft pink

LA TULIPE NOIRE-The nearest to black tulip

.10

.75

6.00

MR. FARCOMBE SANDERS-Fiery rose scarlet

.10

.50

3.50

MRS. KRELAGE-Bright rosy, pink, perfect flower

NORA WARE-Very fine heliotrope

REV. EWBANK-Lavender violet......................

WHISTLER-Rich blood red..

.10

.10

WM. COPELAND-Bright violet

.10

BARTIGON-Carmine red

.10

PROF. RAUENSHOF-Pale rosy red, large..

.10

THE SULTAN-Glossy maroon black

.10

MASSACHUSSETTS-Vivid pink with white center

CENTENNAIRE-Violet rose with blue center

EDMEE

Vivid cherry rose, large flower.

PHYSCHE-Light rose with a pale blue base SIERAAD van FLORA-Fine rose.

YELLOW DARWIN-(Inglescombe Yellow) Mixed Darwin Tulips, a mixture as imported from Holland.

Special Mixture, mixed by us from the foregoing named varieties.

.10

.50

3.50

.65

4.50

.50

3.50

.50

3.50

.60

4.00

.60

4.00

.50

3.50

.50

3.50

.50

3.50

.50

3.50

.10

.10

.10

.10

.10

.50

3.50

.50

3.50

.50

3.00

.50

3.50

.60

4.00

.40

3.00

.60

4.00

\section{BREEDER TULIPS}

Breeder tulips are closer to the parent type of this ancient flower than others. The origin of the tulip is hidden in the dim past. But we know that the Dutch fanciers who in 1634 to 1637 speculated in new varieties during the reign of tulipomania grew the "mother bulbs," or Breeder tulips, to produce new striped varieties by "breaking." The Darwin race is supposed to have developed from the Breeders. For a long time the dark, rich colors of the latter were neglected in favor of the brighter and clearer tones of the other types. But recently taste has changed, and the richly hued Breeders have come into favor.

Though not so tall, perhaps, produce as a rule, even larger blossoms than the Darwins. The colors include many rare among flowers-browns, bronzes, purples, blue-blacks, deep mauves, dull gold and dark lilac all exquisitely blended.

Planting. Plant the same as Darwins. 
ABD-EL-KADER-Copper color....................... $\$ .15$

AESCULAPUS-Clear violet blue..................... $\quad .25$

AURORA-Large violet-rose ............................ . .25

BACCHUS-Dark violet ..................................... .10

APRICOT-Apricot, flushed orange.................. . .15

ASPASIA-Red, bold flower.

BRONZE KING-Bronze and yellow.

BRONZE QUEEN-Tan and apricot.

CAESER-(Clown), light blue.

CARDINAL MANNING-Wine red, flushed brown

CHESTER HUNT-Sky blue; tall

COPERNICUS-Dark coppery bronze and old rose.

DON PEDRO-Coffee brown, shaded maroon GOLDEN BRONZE-Old gold and bronze.... GOLDFINCH-Tortoise color, bronze and yellow

JANE D'OEUF - Rose lilac, broadly edged LOUIS XIV-Dark purple golden brown........ LUCIFER-A bold fiery orange. MARYLAND-Orange brown with light edge MADRAS-Dark bronzy yellow and old gold yellow

MEDEA-Rosy carmine, tinted salmon.......... PANORAMA-(Fairy) reddish mahogany.... ROI SOLEII-Dark violet blue with bronze ST. JAMES-Old rose, bronzed edged............. SAPPHO-Dark purple violet.

YELLOW PERFECTION-Olive yellow color MIXED BREEDER TULIPS.
Doz.

$\$ 1.00$

2.00

2.00

1.50

1.25

.75

.75

.75

.75

.10

.25

.80

.2 .00

1.25

1.50

.85

2.00

.25

1.25

2.00

6.00

.75

1.00

.75

.75

5.00

1.00

5.00

1.00

.75
1.00

$\$ 7.50$

15.00

15.00

10.00

9.00

5.00

5.00

5.00

5.00

5.00

15.00

9.00

10.50

6.00

15.00

9.00

15.00

45.00

5.00

7.50

5.00

5.00

30.00

7.50

30.00

7.50

5.00

\section{SPECIAL MIXTURE OF LONG STEMMED TULIPS}

Doz.

A complete collection of all colors of long stemmed tulips made of named varieties listed in this catalog with great care, insuring a wonderful assortment for your garden

\section{COLLECTION OF TULIPS}

One each of 25 varieties, all different, packed in a neat box. 


\section{The Greatest of Tulips is Louis XIV YOU CAN BUY 12 THIS YEAR FOR $\$ 1.25$}

Few will dispute the claim of Louis XIV to first rank among the tulips. It may have a rival in height, though it would be difficult to find one. There may be other varieties as large, and some which compare with it in rich and beautiful coloring. But to match it in all three respects, in dignified height, impressive size and color all combined, there is no variety that we know. By general consent, Louis XIV has reigned king of all tulips, since it was introduced as a new variety some 12 years ago.

It was priced at $\$ 13.00$ a dozen. The price of a tulip is not governed entirely by merit. The fact that there are relatively few bulbs in existence during the first years of its propagation, forces a high price. As the number of bulbs increases this price naturally falls, without any corresponding decline in the merit of the flower. It has been so with Louis XIV, but the drop has not been rapid. In 1926 it was catalogued at $\$ 3.00$ a dozen, which was less than some of the newer sorts of far less merit.

This year the large Dutch production gave us a chance to buy a quantity of Louis XIV at a price which would permit us to make a special offer to our customers, and we resolved to do what we could to place Louis XIV bulbs in as many gardens as possible. We offer first quality bulbs of Louis XIV for $\$ 1.25$ a dozen; and we urge everyone who has a favorable place to grow them, to invest in at least a dozen of these finest of all tulips. And it may be a century before any better tulip is developed than this truly royal flower.

The rich coloring of this variety is at its best when planted with flowers of a lighter color, and in a position which enjoys the full sunlight. Louis XIV makes a wonderful cut flower, and is often used with petals turned back, to show its beautiful center.

\section{REMBRANT TULIPS}

Rembrandt tulips are in reality "broken" forms of Darwin Tulips. Broken tulips, on account of their odd colorings and variegations, were very popular at one time, and while the modern tendency to favor self-colors is recognized generally throughout all countries, these broken forms passess splendid value. The stems average 18 to 22 inches in height.

EXTRA FINE MIXED 


\section{COTTAGE TULIPS}

As with the Darwin and Breeder types, the classification of Cottage tulips is by race and history, rather than any striking difference in appearance. This type is inclined to have pointed petals, sometimes turned back, and the blossom to be smaller and more slender. But they harmonize perfectly with the Darwins and Breeders and supply a quality of clear, bright color which these lack; while they have pure yellows not found in the others.

Every known shade is to be found among these, from deepest crimson, to bright red, orange, glorious yellows, pink and a host of delicate tints of yellow, pink, cream and heliotrope. They blossom a few days later than the Darwins, often carrying the flowering period of Spring bulbs into the early days of June, especially if planted in a shady position. Many sweet-scented varieties are included, such as Ges. Lutea.

GESNERIANA MAJOR-Most brilliant scar-

let, blue center.

GESNERIANA LUTEA-Tall yellow.

INGLESCOMBE PINK-Even colored pink..

INGLESCOMBE YELLOW-Fine yellow.

ORANGE KING-Glowing orange.

MIXED MAY FLOWERING TULIPS

Ea. Doz. $\$ .10$ .10 .10 .10 .10

\section{MENDEL TULIPS}

An entirely new strain of tulips, raised from crossings between Duc von Tholl and Darwin tulips. Most of the acquired varieties produce flowers of the Darwin type but with colors of the early tulip class. Very early.

SUPERFINED MIX. Each, 10c; dozen; $\$ 1.00 ; 100, \$ 6.00$

\section{STRIPED TULIPS}

Though too gay for modern taste to serve in large plantations, the old-fashioned striped tulips are beautiful. They prove that the Dutch tulipomania was not without excuse, and no gardener who wishes his collection to be representative will be without some specimens.

For indoor decoration a few of these truly beautiful and unquestionably striking flowers will be found most welcome. Particularly in May, when they blossom, will their contrasted shades be welcome both indoors and out. Culture same as for Darwins. BIZARRES-Mixed. Flowers beatifully blotched, striped and feathered with crimson, pink, scarlet, rose, lilac, and purple on yellow ground. Per doz., 75c; $100, \$ 5.00 ; 1,000, \$ 45.00$.

BYBLOEMENS Mixed. White ground, blotched, striped or feathered with lilac, purple, violet, blue. Per. doz., 90c; 100, $\$ 6.00$; $1.000, \$ 55.00$ 


\section{LILY FLOWERING TULIPS}

A new class of Tulips very beautiful and decorative. A cross between Darwins and Tulipa Retroflexa. The flowers have the grace and shape of Liliums as the name indicates.

\begin{tabular}{|c|c|c|c|}
\hline & Ea. & Doz. & 100 \\
\hline ADONIS-Rosy-red, tall stem & $\$ .20$ & $\$ 1.25$ & $\$ 9.00$ \\
\hline ARTEMIS-Carmine rose with white base.... & .20 & 1.25 & 9.00 \\
\hline 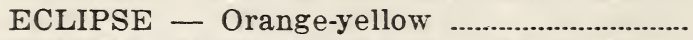 & .60 & 5.00 & 30.00 \\
\hline SIRENE-Satin rose, strong and long stem.. & .20 & 1.25 & 9.00 \\
\hline
\end{tabular}

\section{PARROT TULIPS}

Their fringed and corrugated petals with their wonderful variation make these old-fashioned flowers the most curious of all tulips. Flowering at the same time as other late sorts; they are entirely distinct from any other; the flowers are thick-petaled with extra long stems, and keep extremely well after being cut. The average height is 15 inches.

\section{IRIS}

\section{SPANISH AND HOLLAND IRIS}

(Plant During September)

(FLOWER TIME-APRIL-MAY)

One of the most satisfactory varieties of Iris to grow, being particularly hardy. The flowers are of great beauty, having many different blendings in color, and if cut when in the bud will last a long time in water. For best effect they should be planted in clumps or masses of from 25 to 100 or more bulbs, set 6 inches apart and 2 to 3 inches deep. They grow 18 to 24 inches high and bloom from the end of March on through June.

(These should be planted during September. Orders should be booked early, and we will not supply after September 30th. All orders booked subject to crop production.)

\section{SPANISH IRIS Ea. Doz. 100}

OPHIR D'OR-Good yellow, with fluted

falls, very fine.

$.10 \quad .75 \quad 5.00$

DUBLOON-Fine new yellow, standards

slightly lemon, standards good deep

yellow

.15

1.00

6.00

KING OF THE BLUES-(Count of Nas-

saus). Dark blue

. .10

QUEEN WILHELMINA-Finest white.......... .10

CAJANUS-Late deep yellow.

.10

$\$ .75$

$\$ 5.00$

ROYAL BLUE-Clear blue

.10

.75

5.00

5.00

.75

5.00 
BRITISH QUEEN-White

.10

EXCELSIOR-Light blue

SOLFATERE-Deep blue

.10

FLORA-White with light blue

.10

PRINCE HENRY-Purplish bronze.

.10

LEANDER-Deep yellow

GYPSY QUEEN-Brown and bronze

.10

FORMOSA-Deep blue

\section{IRIS FILIFOLIA IMPERATOR}

The outstanding type in bulbous iris. Very hardy, tall growing, good forcer, immense large blooms of deep blue, fall edged yellow.

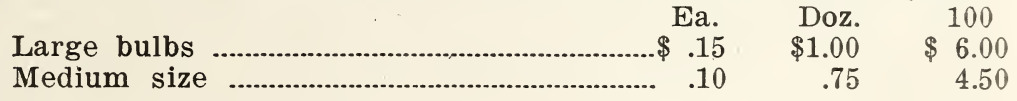

\section{HOLLAND IRIS}

This is an improved Spanish Iris, of greater size, finer colors and blooming several weeks earlier.

REMBRANDT-Dark blue, orange splotch....\$ .10

FRANS HALS-Falls cream, stds. light blue .10

HART NIBRIG-Clear blue.

ALBERT CUYP-Falls bluish white, stds.

primrose

DAVID TENIERS-Lemon yellow and laven-

der blue

J. WEISSENBUCH-A uniform pale mauve

ANTONE MAUVE-Pearl blue.

DAVID BLES-Blue, very early

PIEULMANNS - Blue

FILIFOLIA CELESTIAL-A clear sky blue.. .10
.10

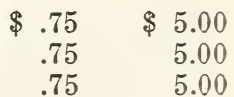

.15

.75

5.00

.10

.10

.10

.10
.75

.75

.75

.75

.75

.75

\section{MIXTURE OF HOLLAND AND SPANISH IRIS}
ALL COLORS IN MIXTURE.
Doz.

\section{SUSIANA MOURNING IRIS}

This is a very beautiful and quaint Persian variety growing about 18 inches tall and blooming in April and May. The large flowers are chocolate light gray veined with a network of chocolate lines. The rhizomes should be set 3 inches deep and 12 inches apart. Stock very limited this season.

Strong roots. Price $\$ 1.00$ each 


\section{IRIS PAVONIA}

The true Peacock Iris from Cape Colony and botanically known as Moraea Glaucopis, Moraea being the African representative of the Iris. While of a fragile nature, this variety is well worth the while in your garden-a wonderful white iris with segments the size of a quarter dollar with a blue spot at the base like a Peacock eye. Stock is very scarce in America.

\section{Strong corms, 15c each, $\$ 1.00$ per dozen}

\section{Introduce Your Garden to Some New Iris}

\section{GERMAN IRIS}

If there were any doubt as to which of the perennials is supreme as a landscape flower, the past season has dispelled it. The improved varieties which make the modern iris seem a diffrent race, were seen in their full effect in thousands of the more advanced gardens in spring; and the verdict was unanimous that of all the pictures possible in the flower garden, that painted by the irises is most brilliant.

What flower can match their wonderful range of color, and at the same time equal their variety of size and form? The only color lacking in the iris, red, is the one least essential in the garden. Of pinks, purples, both red and blue; yellows from pale cream to the deepest gold, it is now possible to obtain varieties of almost any height wanted from 18 inches to 4 feet. There are pure whites low and tall, and the beautifully delicate plicatas-white edged with purple-to highlight groups of the duller colors. There are bronzes and browns, and luminous ruby colors, to glow in the sunshine.

Too long have the newer irises been collectors' flowers. The time has come when they must leave the prosaic surroundings of the collectors' rows, and take their rightful places in the garden, where their beauty may be displayed at its best, set off by beautiful surroundings. Give them a background of cool green; group them with studied care for color-harmony and contrast; light up the duller ones with plentiful white; balance the blue-purples with good yellows, and let the pink varieties supply the warmth which completes an entrancing picture.

There is no longer any reason for delay in acquiring some of the better irises. Prices are down and merit has been established so that investment may be made without risk of error. There will always be the very latest introductions, which will interest chiefly those who take pride in the completeness of their list. Even these, at the higher prices, will usually be found a good investment; since the increase should keep pace with reduction in prices. And certainly that garden will soon be out of date which does not begin 
now to add to its attractions those varieties which established the modern standard of beauty in this race, and have now become plentiful and moderate in price.

Irises dominate the border in the period that follows the tulips and precedes the peonies. Their season continues about one month. Those few perennials which blossom with them cannot compare with the irises in grandeur; so that no garden can afford to do without them.

If there is any flower more easily grown, more gratefully responsive to the gardener's care, we do not know it. And our climate seems to have been made for the hardy varieties; they enjoy our cold winters and our hot summers, and ask only a place in the sun, which is well drained. They may be planted in the midsummer, the autumn or the spring; and they do well in each case.

\section{THE CULTURE OF IRISES}

The main cultural detail for the tall bearded or dry irises is drainage and all the sun possible. They require a summer bake to do their best. They also demand a well limed soil. They need no fertilizing but benefit from an occasional dressing of bone meal worked into the soil or a coating of wood ashes in alternating years with lime; or, better still, a coating of gypsum in the spring shortly after growth starts. Gypsum provides the lime element in the form which best suits irises. Gypsum is also the best known preventative and cure of iris rot. It is almost entirely free from insects and disease pests, the rot and borer both being rare and easy to cope with. Ask for circular.

\section{GERMAN IRIS}

Due to the necessity of replanting our entire iris acreage this season, we are making all the standard varieties shown below at $25 \mathrm{c}$ each; $\$ 2.00$ per $12 ; \$ 10.00$ per 100

8.9 ALCAZAR-S. bluish violet, F. deep purple with bronze veined throat.

7.8 AMAS-(Macranta)-S. rich blue, F. violet.

8.3 ARCHEVEQUE-S. deep violet purple, F. purple.

BLACK PRINCE-S. lavender violet, $\mathbf{F}$. dark velvety violet.

7.5 CAPRICE-Self colored Chinese violet.

CATERINA-Tall branched violet self, very large.

CHERUBIN-S. pale mauve, F. pinkish lilac.

CLARENCE WEDGE-S. helitrope tinged yellow; F, purple red, orange beard.

CRIMSON KING-Early rich claret purple.

DOROTHEA-S. mauve, deeper center, F. soft blue with orange veinings.

COMP di COURACCI. 
ELEGANS-Violet blue and purple.

7.8 ELDORADO-A very distinct and striking blend of bronze, helio trope and violet purple.

8.0 FAIRY-White with iridescent shading of blue.

6.5 GERTRUDE-Deep violet, very fragrant.

7.3 HER MAJESTY-S. lovely pink, F. bright crimson tinged darker.

7.9 IRIS KING-S. golden yellow, F. garnet, edged yellow.

8.6 ISOLINE-Large pink, overlaid with amber.

KHARPUT-Rich dark purple, large flower.

7.8 KOCHI-Claret purple self.

7.9 LORELY - S. light yellow, F. ultramarine blue, bordered cream.

8.2 LOHENGRIN-S. and F. uniform shade of catteyla rose, very large.

7.4 MME. CHERAU-S. white, edged blue, F. large frilled.

MRS. H. DARWIN-S. pure white, F. slightly reticulated white.

NIEBELUNGEN-S. olive green, F. yellow purple and white.

7.8 ORIFLAME-S. bright lavender violet, $\mathrm{F}$. violet.

OSSIAN-S. yellow, F. light claret red; very showy.

8.8 PALLIDA DALMATICA-Tall pale blue, scented.

8.1 PARC. DE NEUILLY-Well shaped flower of pleroma violet.

PERFECTION-S. light blue, F. dark velvety violet and black, orange beard.

7.2 PRINCESS VICTORIA LOUISE-S. pale yellow, F. rich violet, edged cream.

7.4 QUEEN OF MAY-S. a charming pink, F. purplish lilac.

QUEEN FLAVIA-Best primrose, yellow.

R. C. ROSE-S. yellow and lavender, F. blue and white edge.

\section{SPECIAL OFFERS IN GERIMAN IRIS}

Ten varieties, including Isoline, Comp. d'Couracci, Lohengrin, Pallida, Caterina, Queen Flava and four others, a $\$ 2.50$ value for

Twenty varieties, including Amas, Black Prince, Isoline, Comp. d'Couracci, Lohengrin, Pallida, Caterina, Queen Flava, Mrs. H. Darwin, Niebelungen, P. V. Louise, Perfection, Rhein Nixe, Gertrude, Her Majesty and four others, a $\$ 5.00$ value for

Thirty varieties, including beside the above, Iris King, Mme. Cherau, Tamerlane, Alcazar and other fine varieties

Forty varieties, one each of 40 wonderful high point varieties described in this circular, all labeled, and delivered 
8.4 RHEIN NIXE-S. pure white, F. deep violet blue with white edge.

RUBELLA-S. rose lilac, F. crimson purple.

SAPPHO-Lilac blue.

7.4 TAMERLANE-S. pale violet, F. deep purple.

8.0 VIOLACEA GRANDIFLORA-Clear violet blue pallida.

WALHALLA-S. lavender, F. wine red.

\section{IRIS STYLOSA}

(Midwinter Flowering Iris)

These iris are incorrectly called by some the Peacock Iris and are short stemmed early blooming varieties.

ANGUSTIFOLIA_Dark blue ....................................................... . .25 each

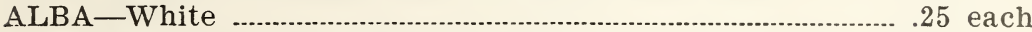

RECENT INTRODUCTIONS OF EXTRAORDINARY MERIT

9.4 AMBASSADOR-40 inches, "S" smoky bronze; F. dark velvety maroon. A strong grower carrying its large flowers well disposed on its stalks; petals of great substance. Vilmorins finest seedling

$\$ 1.00$ each

8.3 AFTERGLOW-Large flowers of soft grayish lavender shading to rich yellow through the center. A thrifty tall grower and free bloomer.

$75 \mathrm{c}$ each

9.1 DOMINION-A wonderful iris. "S" Dauphin's blue or light bluish violet, large erectly held; well balanced and slightly veined; " $F$ " of exceptional substance expanding at the base to a rich indigo-purple velvet. Slight veining at throat; conspicuous orange beard; broad blue green foliage........\$8.00 each

8.7 MILLE. SCHWARTZ-48 inches. Lovely pale mauve flowers of exceptional large size, borne on tall branching stems

$\$ 1.00$ each

9.1 MAGNIFICA-44 inches, " $\mathrm{S}$ " light purple on white; "F" dark reddish purple. Flowers of heavy texture, measuring 6 inches high; stands heat well. Very fragrant. Mid-season....\$1.00 each MOTHER OF PEARL-48 inches. A pale bluish lavender flower of exceptional substance and a lustrous texture. A wonderful iris, tall and vigorous in growth and free flowering. $.50 \mathrm{c}$ each

8.7 OPERA-28 inches, "S" Bishop's (red) purple. "F" purpleviolet. A wonderful coloring.

$50 \mathrm{c} \mathrm{each}$ CECIL MINTURN-24 inches. "S" and " $F$ " a uniform soft shade of cattleya-rose. A beautiful flower, dome shape with broad ruffled petals..............................................................75c each

9.0 LENT. A. WILLIAMSON-42 inches. "S" lavender-violet, "F" velvety royal purple. The flowers are of gigantic size; a tall and luxuriant grower and the flowers are freely produced. 
9.3 SOUVENIR DE MAD. GAUDICHAU- "S" and "F" rich deep purple. Without a doubt the most beautiful of all deep purple iris. The plants are vigorous, the stalks are tall, and the magnificent flowers, of great size, form and substance, are freely produced and well placed. Early.

$\$ 1.25$ each

SUSAN BLISS-42 inches. Exquisite flowers of a uniform shade of deep rose pink, with a light orange beard. Fine shape and of splendid substance. Tall and very free-flowering. One

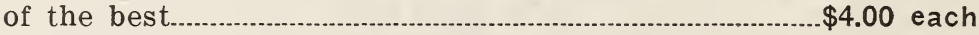
PROSPERO-48 inches. "S" pale lavender flushed yellow at base; "F" deep red purple with lighter shadings at margin. Heavily marked with brown at the halt. Deep orange beard. Vigorous tall growing variety bearing enormous flowers of fine shape $\$ 1.00$ each 8.3 SEMINOLE-30 inches. "S" soft violet rose; "F" rich velvety crismon, brilliant orange beard. An outstanding sort. Midseason $60 \mathrm{c}$ each

\section{IRIS NOVELTIES}

Iris Japonica Fimbriata-It does well in California outdoors in a shady border, and for description let us quote Bailey: "Leaves uniform, thick, smooth, $1 \frac{1 / 2}{2}$ feet long; stem slender, as long as the leaves, with a raceme of lilac flowers; tube $3 / 4$-inch long; outer segments $1 \frac{1}{2}$ inches long with crimped margins, yellow on the claw, crested; finer segments smaller." Introduced from Japan and China. A most wonderful winter blooming Iris.

Strong roots, 50c each; $\$ 5.00$ dozen

Iris Foetidissima (Gladwin Iris) - A native of Central and Southern Europe, England, Afghanistan and Algeria. This Iris is very distinct and is recognized by the odor of the broken leaves. The pods remain on the plants in winter, bursting open and displaying rows of orange-red berries, The flowers are rather inconspicuous, of a lilac shade.

Strong roots, $25 \mathrm{c}$ each; $\$ 2.50$ per dozen

Seed Pods, per dozen stems.

500

Iris Orientalis (Ochroleuca)-One of the largest of the Irises, growing in almost any location. Flower stems rise high above the grass-like foliage, bearing several heads of flowers; yellow, paler or white toward the margin; innner segments lemon yellow.

$25 \mathrm{c}$ each; 12.50 the dozen

Iris (Orientalis) Aurea-A rich golden yellow, growing to a height of five feet in California. A native of the west Himalayas. Flowers find ready sale in florists' shops.

35 c each; $\$ 3.50$ per dozen 


\section{HARDY LILIES}

Lilies are so beautiful, so fragrant, we are likely to think them too fine for the amateur to grow. But many are hardy, thrifty flowers, capable of surviving conditions which would overcome flowers much less beautiful. Grow them in the border in small groups, with background, where their beauty has a suitable setting for its display. They blossom, as a rule, in midsummer, when fine flowers are at a premium. One of the finest effects in the border is produced by lilies in combination with hardy larkspur.

\section{Lilies Arrive From Japan in November}

Most Lily Bulbs, being of late maturity, are not ready before late November.

\section{Outdoor Culture}

When planting lilies out-of-doors, see that they have a well drained position, and where they will be shaded from very hot sun. Such places can usually be found around the shrub borders, and in the hardy plant border. Many varieties are termed "stemrooters," that is, they develop roots on the stem up to two or three inches from the ground. These roots are voracious feeders, and on them depend greatly the number and size of flowers. When they appear, the plant should receive a top-dressing of good rich soil on which they can feed freely. Spade over the ground thoroughly to a depth of 18 inches before planting lilies and enrich it with bone meal. Plant the bulbs 4 to 6 inches deep, depending on their size and the soil, deeper in light soil. Put some sand under and around the bulbs.

\section{MADONNA LILIES (Lilium Candidum)}

Ready July 1-Plant Early

This is one of the oldest, loveliest and best known lilies. Can only be planted in fall. The flowers, of which there are three to twenty on each stem, are snow-white, with heavy yellow stamens and of the most delightful fragrance. It is hardy and will thrive in any ordinary soil, preferring plenty of light and air. Blooms in the open ground about May 30 th in California.

Large bulbs, 25c each; $\$ 2.50$ per 12

LILIUM PARDLINUM (Native California Tiger)

Rich scarlet and yellow, spotted rich brown.

Strong bulbs, 20c each; $\$ 2.00$ dozen

Ready November 1 -Blooms in August

\section{LILIUM LONGIFLORUM}

Ready July 1-Blooms June 1st

Large trumpet shape, pure white in color and of a delicious fragrance.

Strong bulbs, $25 \mathrm{c}$ each; $\$ 2.50$ per dozen 


\section{LILUM MYRIOPHYLLUM (Regale) \\ Ready in November-Blooms in July}

A new and very rare lily from China. The flowers are white, shading to yellow in the center, with a pink tinge on the outer edges. A strong grower, some 3 or 4 feet high. It is deliciously scented, and its extreme hardiness makes it a valuable lily for outdoor planting.

First Size, each, 40c; dozen, $\$ 4.00$

Mammoth Size, each, $75 \mathrm{c}$; dozen, $\$ 7.50$

\section{JAPANESE HARDY LILIES}

Ready December to February-Summer flowering

\section{LILIUM AURATUM}

Ready December

The Gold-Banded Lily of Japan-The most beautiful variety of all the lily family; should be in every garden. Flowers white, dotted crimson, with a clear golden band running through the center of each petal; very fragrant. A splendid lily for pots, or for planting out in the shrub or plant borders. In either way it is very handsome, its large flowers and delicious perfume make it one of the most valuable of all. It is perfectly hardy with ordinary protection and suitable soil.

First Size, (8 to 9 in.), each, $25 \mathrm{c}$; doz. $\$ 2.50 ; 100, \$ 18.00$

\section{AURATUM PLATYPHYLLUM \\ Ready December}

This is without a question one of the most wonderful lilies in cultivation. The leaves are very long and broad and the stems attain a height varying from 7 to 10 feet. The flowers are similar in color to Auratum, heavily spotted, but much larger, the petals more overlapping, and of greater substance.

First Size, 8 to 9 in.), each, $35 \mathrm{c}$; doz. $\$ 3.50 ; 100, \$ 25.00$

\section{LILUM SPECIOSUM (Lance-Leaved Lily)}

\section{Ready in December}

These lilies are probably the most popular of all, succeeding equally well in open border or in pots. When fully open, the petals curve gracefully on the flower stem, exposing the beautiful rose and crimson markings, which characterize the Rubrum and Melpomene types.

RUBRUM-White, spotted and rayed rosy red. We consider this the most satisfactory of the Speciosum class.

First Size, 8 to 9 in.), each $25 \mathrm{c}$; doz. $\$ 2.50 ; 100, \$ 18.00$ MELPOMENE-White, spotted and splashed deep crimson.

First Size, (8 to 9 in.), each 25 c; doz. $\$ 2.50 ; 100, \$ 18.00$ 


\section{VARIOUS HARDY LILIES}

HENRYI-A new, rare, and very beautiful lily from China. The flower stalks, 3 to 5 feet high, carry 5 to 8 large flowers each; apricot spotted brown. $\quad$ Each, 35c; dozen, $\$ 3.50 ; 100, \$ 25.00$

TIGRINUM SPLENDENS-Very hardy native lily; orange, spotted purple.

Each, 25c; dozen, $\$ 2.50 ; 100, \$ 18.00$

\section{CALLA LILIES \\ Ready August-For Fall Planting \\ CALLA (Richardia)}

WHITE CALLA LILY (Aethiopica)-A well-known plant of easy culture for winter bloom, and makes a handsome house or window plant. For outdoor planting, plant crown immediately below surface of ground. Requires moisture.

To aid profuse blooming keep dormant from the middle of June until last of September; pot on receipt in good, rich soil, using 6 to 8-inch pot, give light and heat in abundance. Both foliage and flowers are attractive in this desirable winter bloomer.

First Size (11/4 to $1 \frac{1}{2}$ in.) weight, doz. $1 \mathrm{lb}$. Each, $15 \mathrm{c}$; doz., $\$ 1.50$; $100, \$ 10.00$.

Select Size (11/2 to 2 in.), weight, doz., $2 \mathrm{lbs}$. Each, $30 \mathrm{c}$; doz. $\$ 3.00 ; 100, \$ 20.00$ :

Mammoth (2 to $2 \frac{1}{2}$ in.), weight, doz. 3 lbs. Each, $45 \mathrm{c}$; doz., $\$ 4.50$; $100, \$ 35.00$.

DWARF WHITE CALLA (Godfrey)-Almost an everblooming sort. Culture same as Aethiopica.

Strong Bulbs, $25 \mathrm{c}$ each; dozen $\$ 2.50$

ARUM PICTUM-A so-called black calla. An arum of very dark purplish black reminding one of the Jack in the Pulpit.

Blooming size bulbs, $35 \mathrm{c}$ each

SPOTTED LEAF CALLAS

Ready November 1st-For Later Planting

YELLOW CALLA (Calla Elliottiana)

New and beautiful variety; same habit of growth as ordinary white Calla; flowers same size and shape, rich, clear, lustrous golden yellow; foliage dark green, with translucent creamy spots, strong bulbs.

First Size (11/2 to 2 in.). Each $15 \mathrm{c}$; doz., $\$ 2.00 ; 100, \$ 15.00$

Mammoth (2 to $2 \frac{1}{2}$ in.). Each, $25 \mathrm{c}$; doz., $\$ 2.50 ; 100, \$ 18.00$.

Jumbo ( $2 \frac{1}{2}$ in. and up). Each, 30c; doz., $\$ 3.00 ; 100$, $\$ 22.00$.

IVORY WHITE CALLA (Maculata)

Habit and culture identical to Elliottiana. Leaves dagger shape, spotted white. Flower a pure ivory white. Very hardy.

Strong bulbs, each, 25c; 2.50 per dozen 


\section{ANEMONES}

(Spring Flowering)

Plant September to January

The blossoms of the St. Caen anemones resemble poppies, the flowers are beautiful and come in a great variety of dazzling blues and reds. They flower early in the spring and make a wonderful carpet for a bed or border.

Plant the bulbs, after soaking them overnight, 1 inch deep and 6 inches apart.

DE CAEN-Or poppy flowered single mixed

Doz. 100

Second size bulbs $\$ .75$

Third size bulbs. .60

.50 $\$ 5.00$ 4.00 3.00

ST. BRIGID-Semi-double (but most strains have some singles)

CHRYSANTHAE FLORA-Petal-like stamens

\section{ANEMONE SEED}

DeCAEN-We offer a choice strain of specially grown cultures of Anemone De Caen. Well balanced, and strong in reds. Plant in October for blooms following spring.

Packet, 25c

\section{AMARYLLIS}

Plant July-December-Flower lote summer)

Ea. Doz.

100

BELLADONNA MAJOR-The beautiful rose

pink, early. Bulbs should not be plant-

ed too deep..................................................

BELLADONNA MINOR-Later than Major,

smaller flowers but deeper pink

$\begin{array}{lll}.15 & 1.50 \quad 10.00\end{array}$

wonderful of all the amaryllis family,

sometimes called the Mexican Amaryllis.

Brilliant scarlet, large flower NERINE SARNIENSIS-See under Nerine.

\section{ALSTROMERIA \\ (Peruvian Lily) \\ AURANTIACA-}

A variety of Chilean Lily with spikes of lily-like flowers which are orange or yellow spotted red. A fine cut flower of long lasting qualities. Open exposure plant 6 inches deep. Plant in December, bloom in summer. Rich yellow orange.

Strong bulbs, 25c each, $\$ 2.50$ per 12 


\section{RANUNCULUS}

Nothing produces a brighter or more gorgeous effect in spring than beds of Ranunculus; the flowers are all good for cutting, and the culture is very simple. They thrive in any ordinary garden soil, but should have protection in spring from the late frosts and sharp winds. Plant outside later end of March or early April, as soon as frost is out of the ground, or earlier in the hot bed if desired for early cutting. Plant in November for early spring bloom in frames. Set the tubers 3 to 4 inches apart, pressing them firmly into the soil, claws downwards, and cover them with sand, then with soil; keep the crown of the tubers two inches under the surface. During dry weather the plants should be given abundance of water.

\begin{tabular}{|c|c|c|c|}
\hline & Ea. & Doz. & 1.00 \\
\hline $\begin{array}{l}\text { IEROINE-Tall growing Persian type, rich } \\
\text { butter yellow with light red stripes........... }\end{array}$ & & $\$ .50$ & $\$ 4.00$ \\
\hline ROMANO - Turban type bright scarlet. & & & 600 \\
\hline RENCH MIXED-Large bulbs.............................. & .10 & .75 & $\begin{array}{l}0.00 \\
4.50\end{array}$ \\
\hline ENCH MIXED-Medium size............................. & .10 & .40 & 3.00 \\
\hline RENCH MIXED-Tecolote strain . & .10 & .40 & 3.00 \\
\hline
\end{tabular}

NOVELTIES

FLORENTINE-Large flowers often with white center, colors running more to orange and pink shades. Remarkable for their great size and quality.

$\begin{array}{lll}.10 & .60 & 4.50\end{array}$

PASTELS-Similar to the Tecolote strain in color. Flowers smaller than Florentine, edge of petals frilled distinguishing them from others, and are quite double...........
EGGLESTON-An Australian strain very similar to Tecolote, except that bulb is much longer

$\begin{array}{lll}.10 & .60 & 4.50\end{array}$

\section{AGAPANTHUS UMBELLATUS}

(Lily of the Nile)

While really a plant, it is often listed under bulbs. The flowers are bright blue, produced in umbels of 20 to 30 on stalks two to three feet tall. A long season bloomer. Evergreen the year round, and makes enormous plants. Can also be utilized for tubs.

Strong single divisions, $50 \mathrm{c}$ each, $\$ 5.00$ per 12 , postpaid.

Larger divisions, $\$ 1.00$ and $\$ 2.50$, with delivery charges extra. 


\section{FREESIAS}

(For delivery until October 1st)

Although the culture of Freesias is simple and easy, there is a drawback in that the bulbs have a habit of going on strike. You may plant a nice young bulb which looks the picture of health, which for a whole twelve months gives no signs of life and then starts to grow as if nothing had happened.

The variety most known is the white Freesia Purity, the one grown at Santa Cruz for commercial purposes. We are offering this season some decided acquisitions of merit, with strong branching stems.

PURITY-Finest and purest white variety, large flower and of strong growth.

Doz. 100

BUTTERCUP--Large yellow with deep throat. $\$ .30$ $\$ 2.00$ SPLENDENS-Large flower, lavender violet, strong

grower .60

CALIFORNIA-Large bright golden yellow

CARMENCITA-Light orange salmon hue with buff shading on lower petals.

AURORA-Large flowering yellow suffused with orange

CARRIE BUDAU-Lavender pink, improved General Pershing

ILENA-Rosy lavender with white throat, deep orange blotch on lower petals.

JUNE MICHELSON-Deep rose pink with blush throat, three lower petals are veined purplish crimson with deep yellow blotch on center petal. Good stiff stem

OLIVETTE-Bright carmine red with light yellow throat, lower petals having.deep yellow throat with crimson veins

\section{GLOXINIA}

Ready for shipment Jan. and Feb.

One of our handsomest summer blooming, tuberous-rooted plants. It makes a very fine pot plant, requiring about the same cultural directions as Begonia. One bulb to a 4-inch pot. The colors range through pink, scarlet, violet and white bordered. We are able to offer them in mixture only. $45 \mathrm{c}$ each, $\$ 4.50$ per dozen. 


\section{TUBEROUS ROOTED BEGONIAS}

(January delivery)

Best known as pot plants, but may be used outdoors in plunged pots. Tubers are good for several years.

Rose, white, yellow, orange, scarlet and crimson.

SINGLE-Separate colors or mixed, 25c each, $\$ 2.50$ per 12 .

DOUBLE-Separate colors or mixed, 35c each, \$3.50 per 12.

\section{BABIANA}

A curious gentian blue flower blooming in May, with crocus-like flowers. Plant 3 inches apart and 2 inches deep.

Price per dozen 50c; per 100, $\$ 4.00$

\section{IXIAS}

Plant in October, bloom in May

The Ixia is a beautiful little winter-flowering bulb, with long, slender, graceful spikes of bloom. The colors are rich, varied and beautiful, the center always differing in color from the other parts of the flower, so that the blossoms, expanding in the sun's rays, present a picture of gorgeous beauty. Very desirable for pots.

ENGLISHTON-Wondrous rose Ea. Mixture of many colors $\$ .10$

\section{MUSCARI (Grape Hyacinth)}

Beautiful little flowers for planting in masses either in shade or sun. Once planted, they multiply rapidly and send up flower stems by the score each year. Also adapted for growing indoors. Plant six bulbs in a five-inch pot. Perfectly hardy.

HEAVENLY BLUE-The best blue. Each, 10c; dozen, 40c; 100,

$\$ 3.00 ; 1000, \$ 25.00$.

\section{LEUCOJUM VERNUM}

(Spring Snow flake)

Pretty bulbous plants allied to the Snowdrop, but of much stronger and bolder habit, growing in rich soil from 1 to 2 feet high, and producing freely beautiful large white flowers, distinctly tipped with green; excellent for cutting. When once planted, they take care of themselves. The first year, however, a good mulch should be given so as to protect from freezing, bulbs being Californiagrown. Should have a place in all hardy collections, particularly shady nooks; can be grown in pots.

Each, 10c; dozen, 75c; 100, $\$ 4.50$ 


\section{MONTBRETIAS}

These fine varieties of Montbretias are so easily grown and the results ordinarily obtained so gratifying that we take great pleasure in recommending them to our customers.

Plant during December, January, February and March, they will produce illowers during July and August. They are nearly evergreen and make wonderful cut flowers. Each bulb produces several fine spikes of flowers 20 to 36 inches in height. Use the same culture as for Gladioli.

ROSEA-(New) Rose pink-20c each, $\$ 2.00$ dozen.

KING EDMUND-A beautiful golden yellow. $15 \mathrm{c}$ each, $\$ 1.50$ per dozen.

IMPROVED AURANTICA-A large flower of magnificent orange yellow. Grows tall. One of the very finest. $15 \mathrm{c}$ each, $\$ 1.25$ per dozen.

GERMANIA-A wonderful shade of scarlet orange, very showy. 15 c each, $\$ 1.25$ per dozen.

FIRE KING-Like its name, fiery scarlet red. $15 \mathrm{c}$ each, $\$ 1.25 \mathrm{per}$ dozen.

CREAM-Light tan in color, large flower, remindful one of a freesia, closely following the freesia in blooming season. $15 \mathrm{c}$ each, $\$ 1.00$ dozen.

MIXED-This mixture contains eight separate colors of the finest. $10 \mathrm{c}$ each, $\$ 1.00$ per dozen.

\section{OXALIS}

Most of these Oxalis flower from October until Christmas, and later. Easily grown, requiring only a good, somewhat peaty soil, with considerable light, for their successful growth. Dozen and 100 prices include postage.

GRAND DUCHESS-Lavender, pink and white, doz., 30c; 100, $\$ 2.00$.

BERMUDA BUTTERCUP-Per doz. 30c; 100, $\$ 2.00$.

HIRTA-From the Cape. Forms small plants bearing deep rose colored flowers. Very showy. Form large clumps of tubers on roots. Each, 10c; dozen, 60c; $100, \$ 4.50$.

\section{NERINES}

The so-called Spider or Guernsey Lily. Has the appearance of a miniature amaryllis; stems 6 to 18 inches; umbels of 10 to 12 . Plant September to December 6 inches apart and 4 inches deep. S.IRNIENSIS-Bright crimson, the earliest of all Nerines. Strong bulbs, 25c each; $\$ 2.50$ per dozen. 


\section{TRITONIA}

We have always listed this bulb under LAPEYROUSIA, but other seed stores list it under TRITONIA. It is often called the flame-colored freesia or the pink freesia.

Strong bulbs

Doz. 100

$\$ .50 \$ 3.75$

\section{TRITELEIA}

Grown as pot plant or in borders. Flowers 1 to $1 \frac{1 / 2}{2}$ diameter, egments violet streaked through the center. Hardy. Native of Argentine.

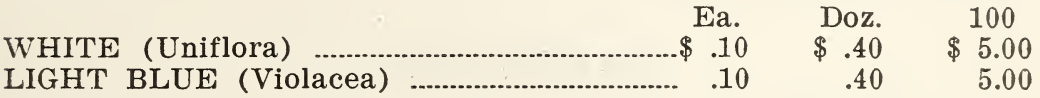

\section{WATSONIA}

Plant September-October-Blossoms May to Jply

Watsonias are similar to gladioli and are in bloom from May on. The spikes grow from 3 to 4 feet tall, and bear many stalks of gladioli-like flowers. They thrive best in a sunny location. Plant the bulbs 3 inches deep and 8 inches apart.

ARDERNEI-The largest and best white.... $\$ .10$ CLEMENTINA - Pink

.20

MRS. ELLA BIRNEY-Red

.20

SALMON PINK-Cerise

.20

LAVENDER

.20

Doz. 100 $\$ .75 \$ 4.50$

2.00

2.00

1.00

12.00

2.00

6.00

12.00

\section{NEW VARIETIES OF WATSONIA}

Ea.

Doz.

100

AMERICA-Delicate lavender pink.

$\$ .35$

CREAM CUP_Creamy white, medium tall.... .35

ESTRELLA-White, outside deep cream, tall .35

EUNICE-Light salmon pink, very lovely......

ED. STURTEVAN'T-Brilliant orange.

HAZEL - Orange pink

J. J. DEAN-Rosepink with deeper shadings

LILLIAN BENNER-Rosy mauve, tall varie-

$$
\text { ty }
$$

SANTA CRUZ-Deep rose.

VICTORY-Deep salmon red.
.35

.35
$\$ 3.50$

3.50

3.50

3.50

3.50

3.50

3.50

3.50

3.50

3.50
$\$ 18.00$

18.00

18.00

18.00

18.00

18.00

18.00

.35

.35

.35

\section{NEW HYBRIDS}

Fine Mixture of all colors, in shades of salmon, lavender, rose. orange, and new white. Doz. $\$ 2.00 ; 100, \$ 15.00$. 


\section{LAPEYROUSIA}

A so-called Tritonia, or flame-colored freesia. 50c dozen, per $100, \$ 3.75$.

\section{ORNITHOGALUM}

(Star of Bethlehem)

A dwarf, hardy, bulbous plant bearing umbels of green and white flowers in April and May. Plant six inches apart, four inches deep, from September to November. Strong bulbs, 15c each, $\$ 1.00$ per dozen; $\$ 4.50$ per 100 .

\section{SPARAXIS}

Plant October-December-Blossom March to April

Beautiful flowers borne on graceful spikes; colors of the brightest shades. They are tigered, blotched, spotted, streaked and flushed. Plant like baby gladioli.

TRI-COLOR

Ea. $\$ .10$
Doz. $\$ .50$
100 $\$ 3.75$

\section{ZEPHYRANTHES}

(Summer Crocus)

Fairy Lilies

Wonderful little edging plants, with decorative grass-like leaves; growing about a foot high and bearing a lovely flower about two inches across. The bulbs multiply rapidly and bloom profusely.

Doz.

100

CANDIDA-Pure white 


\section{FLOWER SEEDS \\ ANTIRRHINUM (Snapdragon)}

For gorgeous coloring few flowers can match Snapdragons. The flowers are of very large size, very fragrant and are produced on immense long spikes, which render them extremely well adapted for cut-flowers. They are easily raised from seed in any rich sunny bed. If intended for winter flowering inside, cut back in September.

\section{TALL MAXIMUM (Giant Flowered)}

These are a wonderful advance in size over all other varieties, each bloom measuring 2 to 3 inches across.

COPPER KING-Velvety copper-scarlet.

GOLDEN QUEEN-Bright deep golden-yellow with lilac-rose throat. PURPLE KING-Rich purple.

THE ROSE-Rose pink.

WALLFLOWER-Brownish orange and copper-red shades. SNOWFLAKE-Pure snow-white, yellow tube.

MAXIMUM (or Giant Flowered), MIXED-All the above and others.

Each of above, pkt. 15c.

\section{TALL LARGE FLOWERING-24 to 36 INCHES}

APPLEBLOSSOM-A charming pink shade on a white ground, with a white throat.

BRILLIANT-Rose.

CORAL ROSE-Dark rose.

GOLDEN KING-Yellow.

ROSE QUEEN-Brilliant rose pink.

MAJUS GRANDIFLORUM MIXED.

Each of above, pkt. $15 \mathrm{c}$.

\section{SEMI-TALL SORTS-18 to 20 INCHES}

Are particularly good for bedding, as they require no staking. The flowers are most wonderful, in many new shades and tints.

GLORIA-Rich deep rose.

GOLDEN QUEEN-Rich yellow.

GRENADIER-Vivid orange, white throat, yellow lip.

NELROSE-Salmon-pink.

QUEEN OF THE NGRTH-Regular bushes covered with large white flowers.

SILVER PINK--Delicate rose pink, seemingly covered with a silver sheen.

Each of above, pkt. $15 \mathrm{c}$. 


\section{SPECIAL MIXTURE OF SNAPDRAGONS}

Includes all these any many other charming varieties and a packet will furnish you with a never-ending color combination of long-stemmed cut flowers suitable for home, church or hospital decoration. 1 oz., $\$ 1.50$; $1 / 4$ oz., $40 \mathrm{c} ; 1 / 8$ oz., $25 \mathrm{c}$; pkt., $15 \mathrm{c}$.

\section{ASTERS}

A popular half-hardy annual produced in a great variety of classes, and an almost endless number of colors. The tallest grow about two feet high, and some varieties of dwarfs are not over eight inches high. The best method of culture is to sow the seed in boxes and transplant when large enough to handle. The seed can be sown, however, in the row where the plants are to remain, thinning them from six to twenty inches apart, according to variety.

\section{AMERICAN BEAUTY (Early Flowering)}

Attains a height of 2 to 3 feet of branching habit. Flowers large, borne on long, stout stems. Planted with Crego and Giant Branching will bloom at same time.

White

Rose
Carmine-Rose

Purple

Mixed, all colors
Lavender

September Beauty

\section{AMERICAN BEAUTY (Late Flowering)}

Is similar in type of flower and growth to the wellknown Late Branching Asters, but differs in that all the large blossoms are produced on much longer and heavier stems. The flowers are fully double to the last.

\section{White}

Carmine-Rose (same shade as American Beauty)

Purple-beautiful rich, velvety purple.

Lavender-beautiful, clear lavender.

September Beauty-delicate, soft shell-pink.

Rose-Pink.

Mixed-all colors.

\section{THE KING}

In form the flower is entirely distinct from any other variety; petals somewhat resemble the quilled varieties, but are much larger and broader, those in the center being curled and incurved, completely covering the crown. In shape and size the bloom is round, full and very large.

White

Rose

Crimson

Blackish Blue

Pink

Lavender

Mixed, all colors 


\section{CALIFORNIA DOUBLE GIANT}

A new family of asters produced by a California seed grower and specialist in asters. They combine the robust habit of growth and length of stem that characterizes the Beauty type and the Crego or Ostrich Feather type of flower. A true non-lateral type.
Peach Blossom
Light Blue
Dark Purple
Dark Rose
White

Each of the above, pkt. 15c; 6 for $75 c$

\section{DELPHINIUM}

\section{(Annual Larkspur)}

These handsome Larkspurs are very effective in borders and planted amongst shrubs. The graceful spikes of bloom are much valued for vases. They continue long in bloom. Sow seed in the open border either in early spring or late fall so germination may take place very early in the spring.

\section{STOCK FLOWERED}

Dark Blue

Dark Rose

Flesh Pink

Lilac

Lustrous Carmine (Newport Pink

Sky Blue

White

Each of the above, $1 / 8$ oz., $25 \mathrm{c} ;$ pkt. $15 \mathrm{c}$.

Mixed, all colors, oz. $60 \mathrm{c} ; 1 / 4$ oz. 20 ; pkt., $15 \mathrm{c}$.

\section{GIANT HYACINTH-FLOWERED MIXED}

Derives its name from the striking resemblance of the flowers to a double Hyacinth. Delicate colors. Pkt., 15c.

\section{SPECIAL MIXTURE}

Annual Larkspurs. A charming mixture containing all the bright and delicate shades, makes wonderful cut flower material and should be planted generously.

Oz. $\$ 1.00 ; 1 / 4-\mathrm{Oz} .30 \mathrm{c} ;$ pkt. $15 \mathrm{c}$

\section{MARIGOLD (Tagetes)}

In late summer, when many bedding plants are past their prime, Marigolds afford a wealth of color that is simply invaluable. The African varieties produce large self-colored blossoms on tall plants; the French are smaller, but the colors and markings are very interesting, some of the varieties being elegantly striped and spotted.

\section{AFRICAN (Fistulosa Type)}

African Tall Double Orange-Beautiful formed flowers of gigantic size and bright color; height, $2 \frac{1 / 2}{2}$ feet. Pkt. 10c. $1 / 4$ oz. 50c; oz. $\$ 1.50$.

African Tall Double Lemon-Like the above but a pure citrus yellow. Pkt. $10 \mathrm{c}, 1 / 4$ oz. $50 \mathrm{c} ;$ oz. $\$ 1.50$.

African Tall Double Mixed-Pkt. 10c. 


\section{FRENCH}

The flowers are much smaller than the African, but are greatly prized for bedding.

Tall French Single Brown-This fine flower is being grown extensively for florists. It is a combination of beautiful shades of brown and fine for cutting. Pkt. $10 \mathrm{c}, 1 / 4 \mathrm{oz} .25 \mathrm{c}$.

Tall French Mixed-In shades of yellow, brown and tricolors. Height, 2 feet. Plit. 10c; oz. 25c.

Dwarf French Mixed-Like the above but only 10 inches high. Pkt. 10c.

Dwarf French Legion of Honor-The flowers are single and a dark, velvety brown, gracefully surrounded by a distinct gold ring. The compact, bushy plant flowers abundantly and is more lasting than any other annual. Pkt. $10 \mathrm{c} ; 1 / 4-\mathrm{Oz} .25 \mathrm{c}$.

\section{DOUBLE FLOWERING STOCKS}

The Stock is one of the many popular plants used for bedding. For brilliancy and diversity of color, fragrance and duration of bloom it is unsurpassed. Sow the seed in pans or boxes and transplant when large enough to an open sunny location. Be careful to save the weak and delicate plants as these usually throw the best doubles. It is also a good idea to plant rather thickly so that the single can be weeded out as they appear. Stocks are one of our best winter blooming plants. May be planted from August to March for a succession of winter and summer bloom.

\section{STOCKS}

This wonderful new strain of Stocks is of branching habit, making it possible to break long branches of flowers from the main stalk without injuring or destroying the beauty of the plant. The flowers are large and many of them on a stem. The sweet perfume of the Stocks make them most desirable for garden or for vases and the Bismark is the very latest improvement.

BISMARK CHAMOIS-Ivory, tinted rose.....................................Pkt. 25c

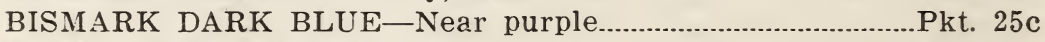
BISMARK BLOOD RED-Bright red............................................... 25c BISMARK GOLDEN BALL-Golden yellow..................................... 25c BISMARK LAVENDER_Light lavender.......................................... 25c BISMARK LILAC-Dark lavender...................................................... 25c BISMARK ROSE-Deep rose pink.............................................Pkt. 25c BISMARK WHITE-Pure white..................................................Pkt. 25c

Collection-One packet each of the above eight separate colors, $\$ 1.50$.

BISMARK MIXED-All colors Pkt. 25c, $1 / 8$ oz. $75 \mathrm{c}$ 


\section{EARLY MAMMOTH BRANCHING NICE}

This type is excellent for bedding and borders and is splendid for cutting. We offer fine varieties in separate colors and a choice mixture. Height 18 inches.

GIANT CARMINE ROSE (Abundance)-Very large spikes. Pkt. 15 c.

GIANT WHITE AND CARMINE (Almond Blossom)-Large, white flowers shaded with carmine. Pkt. 15c. GIANT OLD ROSE (Belle of Naples)-Pkt. 15c.

GIANT CHAMOIS AND ROSE (Golden Sheaf)-A new shade chamois shaded with rose. Pkt. 15c.

GIANT PALE VIOLET (Parma Violet)-Pkt. 15c.

GIANT SILVERY LILAC (Empress Augusta Victoria)-One of the

best. Pkt. $15 \mathrm{c}$.

GIANT CANARY YELLOW (Monte Carlo)-Pkt. 15c.

GIANT FLESH PINK (Beauty Blanc)-Pkt. 15c.

GIANT DARK BLUE (Summer Night)-Pkt. 15c.

GIANT ROSY LILAC (Queen Night)-Pkt. 15c.

GIANT BRIGHT VIOLET-Rich violet or purple. Pkt. $15 \mathrm{c}$.

GIANT NICE-Finest Mixed. Pkt. 15c. 1/8 oz. $75 \mathrm{c}$.

\section{WINTER FLOWERING SWEET PEAS}

Classed as Early Flowering Sweet Peas, for if planted in August or September they may be brought into bloom in the late Fall and early Winter. The planting season in California is from the first of August until the last of January. The varieties selected have long stems with three to four blooms to the stem.

(25 seeds in the packet)

EARLY ALL WHITE-Largest waved pure white.

EARLY AMETHYST-Light purple, very fine.

EARLY AVIATOR-Dazzling crimson scarlet.

EARLY BLUE BIRD-Violet blue, extra large.

EARLY COLUMBIA-Salmon rose, standard with white wings

(Early Blanche Ferry.)

EARLY CREAM-Immense frilled blossoms.

EARLY GLITTERS-Standard bright fiery orange, wings deep orange.

EARLY HARMONY-Finest lavender.

EARLY HERCULES-Extra large florist pink.

EARLY ILLUMINATION-Salmon cerise.

EARLY LAVENDER KING-A new lavender.

EARLY ROSE QUEEN-Rose-pink.

EARLY IRENE-Lavender George Herbert.

EARLY ROSE DORE-Rose pink suffused orange.

Each of the above, pkt. $15 \mathrm{c}, 1 / 2$ oz. $45 \mathrm{c}$ 


\section{NEW INTRODUCTIONS}

EARLY GRENADIER-Dazzling scarlet.

EARLY MRS. KERR-Salmon. A sensation.

EARLY NEW BLUE-Deep blue extra large.

EARLY ORANGE KING-Glowing intense orange.

EARLY STARLIGH'T-Clear lavender blue.

EARLY SWEET LAVENDER-Pure lavender self.

Each of the above, pkt. $25 \mathrm{c}, 1 / 2$ oz. $75 \mathrm{c}$

\section{$\$ 1$ COLLECTION}

\section{EARLY FLOWERING SPENCER SWEET PEAS}

Rainbow Collection of Early Flowering Spencer Sweet Peas, 1 pkt. each of 10 varieties noted below for $\$ 1.00$.

Lavender-Sweet lavender; Rose-Hercules; OrangeOrange King; Pink and White-Columbia; White-All White; Blue-New blue; Red-Aviator; Cerise-Glitters; Rose Pink -Rose Dore; Purple-Amethyst.

EARLY FLOWERING ORCHID MIXED-A well balanced mixture of many fine varieties including some of the late introductions and improved strains. This mixture is just as good as are the named sorts. Plant one ounce to a 30 foot row. Pkt. $10 \mathrm{c}, 1 / 2 \mathrm{oz} .40 \mathrm{c}, \mathrm{oz} .60 \mathrm{c}$, $1 / 4$ lb. $\$ 2.00$, lb. $\$ 6.00$.

\section{Butterfly or Spencer Varieties}

The most beautiful blossom in shape, size, color and fragrance, produced so abundantly and long on lightsome, free-hearted, happy plants, Its flowers dance and sparkle in the sunlight the whole summer through, ever changing, yet ever beautiful. You can cut armfuls of flowers yet it smiles and pursues its beneficient way. It is easy to grow Sweet Peas, because they fairly spring into radiant life in the hands of those who love them.

APPLEBLOSSOM-Standard rose, showing veins of deeper rose,

wings light carmine, the whole color overspreads on primrose. AUSTIN FREDERICK IMPROVED-A new lavender.

BLUE PICOTEE-White, edged violet-blue.

COMMANDER GODSALL-New, violet-blue.

CONSTANCE HINTON-Of enormous size. The best white for outdoor planting.

CRIMSON KING-A rich deep crimson, the best red, does not burn. DEFINANCE-A fine large orange-scarlet. 
DOBBIE'S LAVENDER GEO. HERBERT - Improved Florence Nightingale, opens a little mauvish, but clears to pure lavender. ELFRIDA PEARSON-Large deep blush waved buds and young flowers having a bronze sheen.

FIERY CROSS-A new shade of Turkey-red or military red.

GEORGE SHAWYER-Giant salmon-rose.

GIANT ATTRACTION-Fawn-pink, cream ground.

GOLDEN GLORY - Glowing orange.

HAWLMARK PINK-A wonderful shade.

HEBE-Large bright pink.

ILLUMINATOR-Bright salmon, cerise sparkling with orange.

KING EDWARD VII-Rich waved crimson.

KING WHITE--Produces gigantic flowers absolutely pure white.

MAJESTIC CREAM-Rich deep cream.

MARGARET ATLEE-Apricot pink on cream ground.

MASTERPIECE-Clear lavender self, slightly flushed rose on standard.

MISS CALIFORNIA-Orange-salmon with cream-pink.

PICTURE-Flesh pink suffused creamy apricot.

MRS. TOM JONES-Bright delphinium-blue, a very attractive color. PRESIDENT HARDING-Peach-red, a new shade.

ROYAL PURPLE-The best purple.

ROYAL SCOT-The most brilliant scarlet. It does not fade or burn in the hottest sunshine.

TANGERINE-Deep orange-colored flower, almost approaching the color of a tangerine orange.

THE CARDINAL-Brilliant poppy-scarlet.

WARRIOR-Rich chocolate-maroon.

YOUTH-Large white, pink picotee edge.

Each of the above, pkt. 10c; oz. $40 \mathrm{c}$

\section{Special Mixture}

Spencer Varieties. This mixture is one we make ourselves from named sorts of late flowering ruffled or Spencer varieties and a few of the early flowering ruffled. The result will be a gorgeous display of wonderful blossoms for a long period of time if cultural directions are followed in planting seed and subsequent care in watering.

Pkt. $10 \mathrm{c} ;$ oz $30 \mathrm{c} ; \mathrm{I} / 4$ Ib. $\$ 1.00 ;$ Ib. $\$ 3.00$

\section{Later Introductions}

HEAVENLY BLUE-A new delphinium blue, pkt. 15c.

MARY PICKFORD-Dainty cream pink suffused salmon, pkt. $15 \mathrm{c}$. WEMBLEY-Lavender suffused plumbago blue, pkt. 25c. PINK PERFEC'TION-Rose pink cream ground, pkt. 25c. 


\section{BEST SWEET PEAS}

Each year this collection is revised, newer varieties being included as the older ones are dropped.

Price, one (regular size) packet of each for $75 \mathrm{c}$. If purchased separately would cost $\$ 1.00$. We cannot make any change in varieties.

White Dark Blue

Salmon Pink Maroon

Crimson Picotee

Blue (light) King White

Lavender Hawlmark Pink

Cream Pink

Orange
Crimson King

Mrs. Tom Jones
Austin Frederick Improved

Picture

George Shawyer

Warrior

Commander Godsall

Youth

\section{DAHLIA FLOWERED ZINNIAS}

By F. C. McNabb in Western Homes and Gardens

Dahlia Flowered Zinnias were originated in Southern California. They are different from the Giant Zinnias in the following respects; Petals are cupped, flowers are cupped. Shades are very pleasing and the flowers are extremely large. They are a sensation wherever they are shown.

The Giants and California Zinnias are very similar except that the petals are over-lapping and the centers are high. Flowers are extremely large but not quite so large as the Dahlia Flowered Zinnias. Flowers in both types are extremely doubled.

Seeds should be planted after the soil is warm. Cold soil will decay them. They are better planted in the open ground and thinned to twelve or fifteen inches apart in the row. The first flowers to open are rather slow in developing. The longer they are left on the plants the larger they will grow. Removing the faded flowers is important, or cutting them when they are full grown. Plants will branch readily when the flowers are cut. It is well to cut with long stems and keep them branching near the ground.

The zinnia is one of the most brilliant and satisfactory of the annuals, and one of the easiest to grow. It may be planted outdoors in California until July or August with a certainty of a fine crop in bloom during the late summer and autumn. It is one of the annuals in which a late start is no great handicap as it will not spring into vigorous growth until the weather is warm. They like rich soil and full sun. Also want a good supply of moisture to do their best and should be liberally watered in spells of drought. 


\section{Bodger's Dahlia Flowered Zinnias}

Indicating the excellence of this Improved Strain of Zinnias John Bodger \& Sons Company were granted an Award of Merit by the Royal Horticultural Society of England, 1924. This wonderful flower creates a sensation wherever it is grown and it has probably gained popularity more quickly than any other improved garden flower offered in recent years. The large size, fullness of petals, extraordinary color range, and good keeping qualities have all contributed to its popularity. The flowers resemble in appearance the Decorative Dahlia and is called the Dahlia Flowered Zinnia for this reason. When in full bloom they often measure five to six inches in diameter and many of our friends who have grown them take the trouble to write to us and even send us pictures of the wonderful flowers they have produced. The Dahlia Flowered Zinnia is strictly a California introduction and since it is so easily grown and is immune from disease at this time we cannot recommend it too highly to our friends. The colors do not always come entirely true but the variations are desirable colors.

\section{Dahlia Flowered, 3 to 4 Feet}

In formation the broad petals are closely imbricated, sometimes seeming almost to be piled one upon another. The flowers often measure 4 inches in depth and 6 to 8 inches in diameter. In general appearance the flowers compare with the Show Dahlias.

130 ILLUMINATION-Similar to Exquisite but a striking selfcolor of deep rose. (Tyrian Rose).

131 CANARY BIRD-A delicate shade of primrose-very large and holds its color well until out of bloom.

132 BUTTERCUP-An immense deep creamy yellow. A very desirable flower, which should be included in every collection.

133 CRIMSON MONARCH-Rich crimson. By far the largest and best of red shades. Flowers often eight inches in diameter.

134 DREAM-A fine, deep lavender, turning to purple (Mallow Purple).

135 EXQUISITE-By far the most pleasing of our collection. Color light rose with center a deep rose. (Tyrian Rose).

136 GOLDEN STATE-A very rich orange-yellow (Cadmium). Yellow in the bud, turning to an attractive orange when in full bloom.

137 OLD ROSE-This is adequately described by its name, as it is of the real old rose shade; it is large, and for charm and beauty we consider it ranks next to Exquisite.

(See next page for other varieties) 
138 ORIOLE-It is an immense orange and gold bi-color, changing slightly as it ages, but at all times worthy of the beautiful bird for which it is named.

139 POLAR BEAR-A very large pure white, the best white yet seen in Zinnias. True Dahlia form.

Each of the above, pkt. 25c; 5 for $\$ 1.00$

Collection-One packet each of the above named varieties of Dahlia Flowered Zinnias, for a most gorgeous display, $\$ 2.00$

\section{Gold Medal Dahlia Flowered Mixture}

This mixture contains all of the zinnia novelties and is a most wonderful collection. It will make an excellent garden show and produce fine flowers for cutting. Pkt. 25c.

\section{GIANT DOUBLE QUILLED ACHIEVEMENT}

158 The petals are partially tubular or quilled giving the flower somewhat the appearance of a cactus dahlia and displaying a rich variety of colors mostly of the warmer shades, such as salmon, old rose, deep rose, lilac, bronze and dark red tones. On the reverse side the petals are light or dark lilac, making a wonderful combination of colors.. The plants are of strong, robust growth and produce an abundance of very large, double flowers often 5 inches in diameter. Pkt., 25c.

\section{GIANT PICOTEE}

A remarkable new and distinct class with beautiful double flowers in orange, flesh, lemon, pink, and cerise, each petal being distinctly tipped with various shades of dark, resembling the Picotee Carnations.

156 GOLDEN PHEASANT-A variety of the above, having flowers of a deep orange with maroon tips. Pkt., 25c.

157 GIANT PICOTEE MIXED. Pkt., 25c.

\section{Small Flowered Zinnias-Haagaena}

160 Of new introduction, drawf, the plant carrying small, prettily marked flowers. A variety when better known will have a big demand. Pkt., 25c.

170 LILLIPUT DOUBLE MIXED-Small double flowers. Dwarf compact bushes and profuse bloomer. A good Zinnia for cutting. Pkt., 25c. 


\section{GRASSES FOR LAWNS}

Grass seed for lawns may be planted in the fall or in the early spring. Use every effort to have ground in best of condition before sowing. Prepare soil well and after sowing the seed sprinkle with bone meal, and rake seed in lightly, and roll down but not too tight. If weather is warm, it is advisable to mulch with straw. Keep wet. Use one pound of seed to 200 square feet except clover, then use one pound to 300 square feet. It pays to plant thick.

Each fall with the first rains, try applying bone meal, blood and wood ashes dusted over your lawn. Regular periods of rolling will improve its appearance.

\section{Fancy Kentucky Bluegrass (Poa Pratensis)}

The greatest of all lawn and pasture grasses for this country. It requires time to establish itself and should be grazed on lightly for the first two years. The seed may be sown in very early Spring or in September. Sow 40 to 60 pounds per acre for pasture and 100 to 135 pounds to the acre for lawns.

EXTRA FANCY SUN CURED SEED-Specially recleaned and of high germination; $1 \mathrm{lb} .50 \mathrm{c} ; 10 \mathrm{lbs} . \$ 4.00 ; 50 \mathrm{lbs} . \$ 18.00 ; 100$ lbs. $\$ 35.00$.

\section{Red Top Grass (Agrostis Alba)}

This grass is well adapted to moist and acid soils but will grow and succeed on any and all kinds of soils. It is one of the most valuable grasses for pastures and meadows but not quite as nutritious as Timothy for hay. Sow in Fall or Spring with or without a nurse crop. To sow an acre of pasture use 8 to 10 pounds of fancy or 20 pounds of virgin Re d Top. It is invaluable in a lawn grass mixture as it comes quickly and protects the slower-growing grasses until they can establish themselves.

Extra Fancy recleaned solid seed, (32 pounds per bu.): 1 pound, $40 \mathrm{c} ; 10$ pounds, $\$ 3.50 ; 50$ pounds, $\$ 15.00 ; 100$ pounds, $\$ 25.00$.

\section{White Clover}

Extra fancy seed. Price, $1 / 2$ pound, $40 \mathrm{c} ; 1$ pound, $75 \mathrm{c} ; 10$ pounds, $\$ 7.00$.

Prices subject to market changes. 


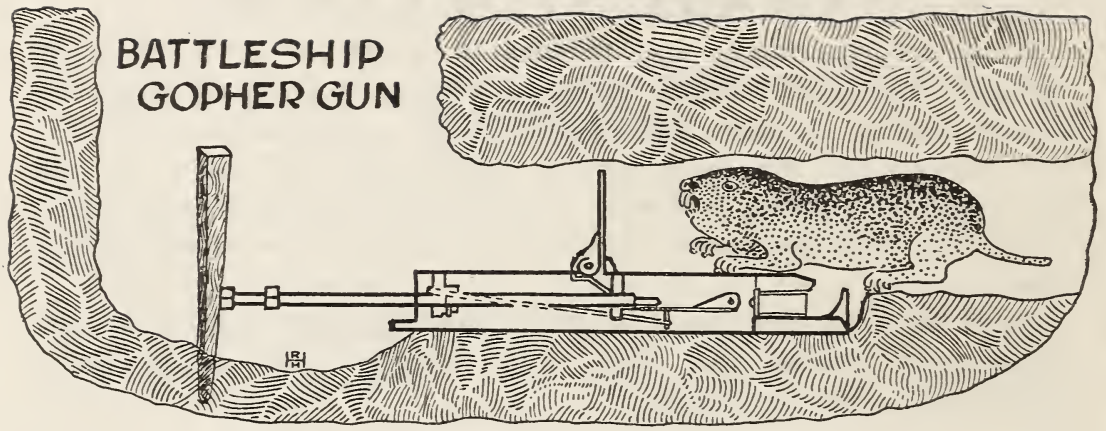

REDUCES THE STAR BOARDER TO A MINIMUM

The simplest gun on the market. Built of highest quality manganese bronze and number one brass. Danger of harm from premature discharge at a minimum. Spring trouble eliminated by use of ordinary $15 / 8$ inch by $1 / 8$ inch rubber band for firing control. Easy swing of the keel permits cleansing of parts, and reduction of back-fire.

The success of "shooting gophers" is positive if the gun is properly placed. Notice in the illustration how the gun should be placed on the level of the run. Try an old table knife to clean out the run and to form a slot to fit the gun.

DO NOT pull out firing pin until gun is in place. Gun does NOT need oiling. Use $.38 \mathrm{~S}$. \& W. blanks.

\section{$\$ 1.75$}

\section{FERTILIZERS}

As prices are dependent on cost of raw products in open markets, price list of fertilizer's will be furnished on request. 


\section{COLOR PLATES}

For the reason that seldom does a color plate do justice to the flower it illustrates, we have purposely refrained from color plates in this catalogue.

However, for educational purposes, by schools and garden clubs, there is a demand for color plates. To meet such a demand we have secured colored plates and offer them as follows:

\section{DARWIN TULIPS AND HYACINTHS}

A six page folder (7 $3-8 \times 11)$ depicting TWELVE DARWIN TULIPS TO BEAUTIFY YOUR HOME GARDEN and FOUR SELECTED HYACINTHS. The front cover shows a bed of tulips alongside a lawn, with inset of three flowers in color. Plans for planting destribed on reverse.

\section{GLADIOLI COLLECTION}

A three page colored folder showing twelve gladioli in color with group of three on the cover. Fourth page gives planting instructions.

\section{IRIS}

A beautiful six page iris plate favoring twelve wondrous iris, with a beautiful cover and back. Blue print plans for favorable plantings.

These may be had in lots of 25 to 100 at $10 \mathrm{c}$ each

JUST THE THING FOR YOUR GARDEN CLUB 


\section{TOO LATE!

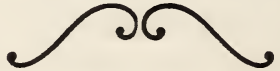

Your Bulb Order Should be

\section{Sent in Today}

It is so easy to postpone placing your bulb order until the season is passed for proper planting

A large proportion of your success with bulbs is due to planting AT THE PROPER TIME

So Check Over This List Now Send it in For Booking Now And the Bulbs Will be Delivered you as Ready and in Proper Time For Planting

How Easy and How Satisfactory

At Christmas, a box of bulbs is always a lasting gift. An acceptable gift to meet you: puise. 



\section{BEGINNERS' BULB OFFERS \\ SPRING BULBS}

Q1 To introduce to you the variety and beauty of $\$ 1$ spring flowering bulbs we will send the following, all of first quality: 6 Scillas, 6 Crocuses, 6 Gladioli, 6 May-flowering bulbs and 6 Narcissi30 bulbs in all-for $\$ 1$, postpaid.

\$5 We will send bulbs of the finest quality of \$5 our selection, all named varieties, or single colors, no mixtures, bagged separately with names on bags-the following assortment: 25 Crocuses,

25 Scillas, 25 Gladioli, 50 May-flowering Tulips, 25 mixed Daffodils, 18 Paper White Narcissus for indoor growing-168 bulbs for $\$ 5$, prepaid.

\$10 We will send bulbs of the finest quality and $\$ 1$ of the better named varieties, bagged separately, with names on bags-the following assortment: 50 Crocuses, 50 Scillas, 25 Gladioli, 50 Darwin Tulips, 25 Cottage Tulips, 25 Breeder Tulips, 45 mixed Daffodils, 30 Paper White Narcissi for indoor growing, 25 Bedding Hyacinths -325 bulbs for $\$ 10$, not prepaid.

\$25 We will send bulbs of the finest quality and $\$ 25$ of the better named varieties, bagged separately, with names on bags, in the following assortment: 150 Crocuses, 150 Scillas, 100 Gladioli, 150 Darwin Tulips, 150 Cottage Tulips, 100 Breeder Tulips, 100 mixed Daffodils, 25 Paper White Narcissi for indoor growing, 25 Bedding Hyacinths-950 bulbs-for $\$ 25$, not prepaid. 